\title{
NONLINEAR WAVE PROPAGATION IN DISORDERED MEDIA
}

\author{
ANGEL SÁNCHEZ and LUIS VÁZQUEZ \\ Departamento de Fisica Teórica I, Facultad de Ciencias Físicas, \\ Universidad Complutense, E-28040 Madrid, Spain
}

Received 15 April 1991

\begin{abstract}
We briefly review the state-of-the-art of research on nonlinear wave propagation in disordered media. The paper is intended to provide the non-specialist reader with a flavor of this active field of physics. Firstly, a general introduction to the subject is made. We describe the basic models and the ways to study disorder in connection with them. Secondly, analytical and numerical techniques suitable for this purpose are outlined. We summarize their features and comment on their respective advantages, drawbacks and applicability conditions. Thirdly, the Nonlinear Klein-Gordon and Schrödinger equations are chosen as specific examples. We collect a number of results that are representative of the phenomena arising from the competition between nonlinearity and disorder. The review is concluded with some remarks on open questions, main current trends and possible further developments.
\end{abstract}

\begin{abstract}
"We have now emerged from the forest of nonlinear mathematics with, I hope, a greater sense of the correspondence between the richness of the mathematics and the complexity of the processes in natural philosophy. As a closing observation I wish to emphasize that complex systems are intrinsically nonlinear and stochastic, perhaps one or the other dominating in a particular situation, but in general both aspects are important for a complete understanding of the system. How one utilizes this insight in a particular context remains to be seen."
\end{abstract}

Bruce J. West, An Essay on the Importance of Being Nonlinear

\section{Introduction}

In spite of the fact that there have been a few previous, isolated pioneering discoveries, the 1960's can be considered the years of the birth of Nonlinear Physics, because its main branches originate from two papers which appeared by the middle of that decade. These seminal discoveries are due to Lorenz (1963), who started the study of chaos, and to Zabusky and Kruskal (1965), who initiated the study of solitons. Solitons, or, more generally, nonlinear waves, is the topic we deal with in this review, and we believe it useful to begin with a short summary of their history. From our personal viewpoint, the evolution of the research on this subject might be divided into three periods. The first would comprise the years between 1965 and the early 1970's, the milestone marking its 
approximate end being the celebrated review by Scott, Chu and McLaughlin (1973). By the time of its publication, not only the study of solitons and soliton-bearing systems had gained a previously refused respectability (see related remarks in Scott, 1990), but also the most important and widely applicable nonlinear equations had been brought in. The second period would span the next decade, which was devoted to develop the main mathematical tools to deal with those equations. Besides, nonlinear models entered a great number of different physical scenarios, specially in Condensed Matter Physics (see, for instance, the Proceedings edited by Longrenn and Scott, 1978; Bishop and Schneider, 1978; Bernasconi and Schneider, 1981). Experiments on solitons began, and their existence was satisfactorily confirmed by the late 1970's (Remoissenet, 1990a, $1990 \mathrm{~b}$ ). All these aspects characterize this second stage as the era in which solitons prove themselves a new and fruitful paradigm in physics (Bishop et al., 1980).

The third and last period is still running, and the interest of researchers in this field has somewhat changed. Despite its importance and interest, the study of exactly solvable models is giving up its protagonist role to the investigation of perturbations of such models, because of the necessity to take into account the unavoidable deviations of real physical systems from ideal ones. Perturbations usually give rise to a drastic modification of the equation features, and more often than not they turn them unsolvable. Hence, several approximate analytical techniques have been proposed, some of which are actually very powerful. In addition, the availability of fast computers has allowed a large amount of numerical work. However, both approaches have achieved their best results when applied in cooperation (a procedure termed "computational synergetics" by Zabusky, 1981). Thus, the activity related to nonlinear wave propagation in disordered media is currently enormous (see the Proceedings edited by Pnevmatikos et al., 1989; Bishop et al., 1989; Barthes and Léon, 1990; Abdullaev et al., 1991) and the subject is in a state of "healthy growth" (Scott, 1990).

It is enough to glance at the indices of the above quoted books to understand that the variety of physical, chemical, biological or technological problems that can be modeled by perturbed nonlinear equations is far more ample than that of unperturbed ones. Indeed, there exists a plethora of possible terms to be included into every equation, each one of them arising from corresponding factors influencing the observed behavior of every realization of a nonlinear model. In this context, it is worth pointing out that not only several different perturbative contributions can be thought of, but also that their intensity usually plays a key role in the model dynamics, which can change dramatically when varying the parameters governing the new terms: even chaotic regimes of nonlinear partial differential equations have been found (a topic that lies outside the scope of this paper; the interested reader is referred to Zettl, 1988, or Reinisch and Fernandez, 1989 , and references therein). This is a hint that, in the presence of strong 
perturbations, one must face a situation that by no means is "the sum of nonlinearity and perturbation", so to speak, but a very distinct thing resulting from a complicated, a priori unpredictable interaction between both constituents of the model. Therefore, the so posed problems are very difficult and, in general, they must be dealt with through rather particular and sophisticated techniques. In this sense, Nonlinear Science is now at the same point where Linear Science was at the middle of this century, when the numerical experiment of Fermi, Pasta and Ulam (1955) shook and broke up the linear view of world based on the principle of superposition and the treatability of deviations from linearity by perturbation theory. We must take advantage of this historical lesson and keep our minds open to the possibility of qualitatively new things happening in this exciting world of nonlinear disordered systems.

\subsection{Aims and structure of the paper}

We have just seen that along its thirty-year history, Nonlinear Physics has attracted very much attention, and has accumulated an accordingly large amount of knowledge. It is easily understandable that we will not try to encompass all of it here. As a first restriction, we will consider only wave propagation and we will not say anything about diffusion problems (for reviews on diffusion in disordered systems see e.g., Havlin and Ben-Avraham, 1987, or Haus and Kehr, 1987). Though sometimes we will mention some results on linear ones, either as a historical introduction or for a better comprehension (for reviews on linear disordered problems see Barabanenkov et al., 1970, Elliot et al., 1974, Lifshitz et al., 1988, or Freylikher and Gredeskul, 1991). Furthermore, we will devote our efforts to classical problems. There are a lot of quantum problems, not only linear, but also nonlinear (see the review on nonlinear condensed matter effects by Makhankov and Fedyanin, 1984), and it is practically impossible to include all of them (a good panoramic view is given in the Special Issue on Disordered Solids, Physics Today, December 1988).

Once we have specified the topics we are not going to deal with, let us turn to the ones we actually review. Even with the above restrictions, the number of works is impressive, most of all in this decade. A quick glimpse at them allows to select as representative examples the Nonlinear Schrodinger (NLS) equation and the family of Nonlinear Klein-Gordon (NKG) equations. Yet, they are not only examples, but they have deserved most of the efforts from researchers of nonlinear phenomena in different fields. Due to their large number of applications, general results obtained in the framework of these systems are useful in many contexts. Even more, they allow the propagation of several types of solitons: both models support topological and envelope solitons, the "most nonlinear", so to speak, of these excitations. For these reasons, we will devote the body of the review to these systems, but the reader should have in mind that properties more or less similar to the ones we will describe have been shown to occur in the majority of nonlinear evolution equations. Finally, we have not 
reviewed to its full extension the experimental aspect of the work on physical systems modeled by these two nonlinear equations. Here and there we have included mentions to some experiments, but we refer the reader to the papers by Remoissenet (1990a, 1990b) for recent surveys, in which a large number of further, updated references is contained.

The remainder of the paper is structured as follows. In the rest of this introductory section, we present a general survey of the basic nonlinear models and models of disorder that have been proposed in the past. In Sec. 2 we review the main analytical and numerical techniques that have been worked out to deal with nonlinear disordered problems. We will comment on their applicability as well as on their advantages and drawbacks. We present this part as a separate section because the procedures we talk about are general, and have not been conceived only for NLS or NKG systems. Sections 3 and 4 are the central body of the review, devoted to NKG and NLS equations, respectively. We try to follow a chronological approach as well as to go from the simplest to the most complicated forms of disorder. We do not go into deep detail of calculations involved to get specific results; rather well, we try to make the meaning of these results comprehensible. The final section contains some short closing remarks and points out several open problems.

We would like to stress that we have not tried to collect all the work that has been done in the past on these two equations. Our aim is to offer a flavor of the kind of computations that are either interesting, either feasible, or both at a time; to this criterion should be added the unavoidable bias imposed by the authors' own interests. The reader to whom this review is directed is a non-specialist who is willing to be introduced to the topic. Nevertheless, we have intended to compile a list of references as comprehensive as possible, to enable him to make further progress in particular aspects; we believe that it can be also useful for people working in the field at present. We want to refer both experts and non-experts to the related reviews by Bass et al. (1988), on solitons under stochastic perturbations, and by Kivshar and Malomed (1989) on dynamics of solitons in nearly integrable systems, that provide another viewpoint and are in some sense complementary to this particular paper. Finally, there is still one more remark to do: it is possible that we have skipped important references due to oblivion or oversight, or that we have misunderstood them. We want to apologize to all our colleagues whose works have not been quoted or have been misquoted for one or other reason.

\subsection{Basic nonlinear models}

After these short historical notes and remarks, two questions arise in a natural way: What are the main, basic nonlinear models? How can we model the influence of disorder on them? Let us try to answer the first of these questions in this subsection; we will deal with the other below. The names of two of the basic 
models have already appeared here: the Nonlinear Klein-Gordon (NKG) and the Nonlinear Schrödinger (NLS) models. Let us begin with the former.

The mathematical expression common to all NKG models is

$$
\phi_{t t}-\phi_{x x}+\frac{d V(\phi)}{d \phi}=0
$$

where, from now on, $\phi \equiv \phi(x, t)$ and the subscripts $t$ and $x$ stand for the corresponding partial derivatives; $V(\phi)$ is some function of $\phi$. The most famous NKG equation is undoubtedly that of the sine-Gordon (sG):

$$
\phi_{t t}-\phi_{x x}+\sin \phi=0
$$

whose introduction can be dated back to the paper by Frenkel and Kontorowa (1939; see also Nabarro, 1967), who were interested in understanding the motion of dislocations in solids. The number of applications of the $\mathrm{sG}$ equation is very large. To mention just one at this point, we will say that it has been very fruitful in Josephson junction electrodynamics, where Eq. (2) describes the propagation of magnetic flux quanta (see, e.g., Barone and Paternó, 1982). Another wellknown NKG system is the so-called $\phi^{4}$ equation:

$$
\phi_{t l}-\phi_{x x}-\phi+\phi^{3}=0 \text {. }
$$

The $\phi^{4}$ equation was named after the highest order term appearing in the Lagrangian from which it can be derived (see below; see also Rajaraman, 1975, or Makhankov, 1978). It was proposed by Aubry $(1975,1976)$ and by Krumhansl and Schrieffer (1975) to describe certain compounds which undergo structural phase transitions. We will close this paragraph with one more NKG equation:

$$
\phi_{t t}-\phi_{x x}+\sin \phi+\alpha \sin \left(\frac{\phi}{2}\right)=0
$$

often referred to in the literature as Double sine-Gordon system (DsG), and found in applications in Nonlinear Optics, for example (Bullough and Caudrey, 1978), and, more often, in quasi-one dimensional magnetic systems (Zvezdin, 1979; Bar'yakhtar et al., 1980).

The other example of typical nonlinear features is the Nonlinear Schrödinger or cubic Schrödinger equation (usually termed in shortened form as NLS), that can be written down as

$$
i \phi_{t}+\frac{1}{2} \phi_{x x}+|\phi|^{2} \phi=0
$$


where now $\phi$ is a complex function of the real $(x, t)$ variables. It serves for general purposes concerning weakly nonlinear systems where the dispersion plays an important role (see one of the possible qualitative derivations of the NLS equation in Bass et al., 1988; another, more general is given in Dodd et al., 1982). This equation has been used to describe a lot of different physical situations in diverse fields, from nonlinear optics (Hasegawa, 1988) to molecules (Davydov, 1985). Even more, the NKG small amplitude excitations can be fairly approximated by the NLS equation (Newell, 1985; Remoissenet, 1986), this being another important application of Eq. (5).

Let us finish this catalog with the remaining archetypal family of equations, related by some very interesting mathematical properties, like that pertaining to the same hierarchy. The most famous of them is the Korteweg-de Vries (KdV) equation,

$$
\phi_{t}+\alpha \phi \phi_{x}+\phi_{x x x}=0
$$

which was first derived by Korteweg and de Vries (1895) to explain the famous phenomenon of the long, solitary wave water vividly described by Scott Russell (1844). The $\mathrm{KdV}$ equation is deeply related to systems experiencing the competition of weak nonlinearity and weak dispersion (Zakharov et al., 1984). This happens, for instance, with waves in nonlinear atomic chain models (Davydov, 1985), where it is also found the following generalization of the $\mathrm{KdV}$ equation:

$$
\phi_{t}+\alpha \phi^{2} \phi_{x}+\phi_{x x x}=0
$$

usually referred to as modified $\mathrm{KdV}$ (mKdV) equation. Equations (6) and (7) are indeed profoundly related: they can be transformed one into each other by means of the Miura transformations (Miura 1968). Moreover, both of them can be derived from the Boussinesq equation (Boussinesq, 1872; see also the books by Whitham, 1974; Dodd et al., 1982),

$$
\phi_{x x}-\phi_{t t}+6\left(\phi^{2}\right)_{x x}+\phi_{x x x x}=0
$$

that describes shallow water waves propagating in both directions; one can reduce it to the $\mathrm{KdV}$ and $\mathrm{mKdV}$ equations restricting propagation of waves to one direction only plus some other simplifications (to be precise, the $\mathrm{mKdV}$ equation is obtained from (8) if the nonlinearity is not quadratic but cubic, see Dodd et al., 1982). Equation (8) is rather general, and it has to do with solitons in one-dimensional solid state physics (Flytzanis et al., 1985; Davydov, 1985), in plasma physics (Whitham, 1974; Makhankov, 1978) and in a number of other weakly nonlinear and dispersive contexts. If this last characteristic is absent, but instead the physics of the problem includes dissipation, then the equation 


$$
\phi_{t}+\phi \phi_{x}-\phi_{x x}=0
$$

turns out. Equation (9) was first studied by Burgers (1974) and it is named after him. These last equations ( $\mathrm{KdV}, \mathrm{mKdV}$, Boussinesq, Burgers) are not too complicated to be nonlinear. Thus, the $\mathrm{KdV}$ equation was the first one to be solved (Gardner et al., 1967) completely by means of the Inverse Spectral Transform (IST, also termed Inverse Scattering Transform), and the Burgers equation can be linearized and subsequently solved through a change of variables (the so called Cole-Hopf transformation, discovered independently by Cole, 1950, and Hopf, 1950). This "easier" character is inherited by equations derived from them by including perturbative terms, that sometimes can even be solved exactly in his turn (see examples in the next section), and, even when this is not so, much information can be obtained about their solutions.

We have summarized a number of nonlinear models that share the characteristic of being continuous, i.e., they are defined on the whole or a part of the plane $(x, t)$. However, it is also very common to be faced with discrete problems, where the space variable is not continuous but takes values on a finite or infinite lattice (discrete space-time problems have been scarcely studied, see Herbst et al., 1985, or Herbst and Ablowitz, 1989a). These discrete problems can arise either on their own or as discretizations, via finite differences schemes (about which we will discuss below) of partial differential equations like the ones we have been reviewing above; often, they can be thought of from both viewpoints. We do not want to make an exhaustive list of them, but instead we will mention a few samples. The first one should be, of course, the Fermi-Pasta-Ulam (FPU) lattice:

$$
\frac{d \phi_{n}}{d t}=F\left(\phi_{n+1}-\phi_{n}\right)-F\left(\phi_{n}-\phi_{n-1}\right),
$$

where the choices of $F$ that they studied in their celebrated experiment (Fermi et al., 1955) were $F=\gamma \phi+\alpha \phi^{2}$ and $F=\gamma \phi+\beta \phi^{3}$. The connection between these choices and the Boussinesq family of equations is remarkable: when the continuum limit of $(10)$ is taken, i.e., the lattice spacing goes to zero and $\phi$ becomes a continuous variable, Eq. (8) is obtained for the quadratic $F$, while the cubic version of Eq. (8) appears for the cubic $F$ (see, e.g., Dodd et al., 1982).

A context in which lattice equations customarily arise is that of electrical transmission lines (Remoissenet 1989, 1990a, 1990b). Probably the most famous one was designed by Hirota and Suzuki (1973) to study a partial difference equation proposed and solved by Toda (1967), whose expression is,

$$
{ }^{\prime} \phi_{n}=\exp \left(\phi_{n+1}-\phi_{n}\right)-\exp \left(\phi_{n}-\phi_{n-1}\right),
$$

and was the first discrete equation to be completely solved by IST. Lastly, we will quote another difference-differential equation, the one of Ablowitz-Ladik (AL) 
(Ablowitz and Ladik, 1975, 1976) given by

$$
i \dot{\phi}=\phi_{n+1}-2 \phi_{n}+\phi_{n-1} \pm \phi_{n}^{*} \phi_{n}\left(\phi_{n+1}+\phi_{n-1}\right)
$$

(the asterisk standing for complex conjugation), which is interesting in its own, because it is solvable by IST too (Ablowitz and Ladik, 1975, 1976). In addition, it illustrates how these problems appear by discretizing partial differential equations: it is a discretization of the NLS equation (5), in fact one with very good properties (Herbst and Ablowitz, 1989b).

It goes without saying that this list of nonlinear equations is very far from complete. We have only quoted the main families of systems, and we have skipped other important equations like the Landau-Lifshitz or the MaxwellBloch, for instance (the reader can find information about them in the book by Dodd et al., 1982, and references therein). Even more, we have restricted ourselves to one-dimensional (1D) problems. In spite of the fact that, though somewhat idealized, 1D equations have a lot to do with physical reality (Mattis, 1981). Many times there is no way to reduce a particular situation to a $1 \mathrm{D}$ equation, and then it must be dealt with by studying a multidimensional equation. The knowledge that has been achieved up to know on this kind of systems is rather poor, though very recently this field is undergoing a sudden increase of activity. Examples have been studied mainly in two dimensions, like the Davey-Stewartson (kind of a generalization of the NLS problem, see Davey and Stewartson, 1974, and Anker and Freeman, 1978) or the KadomtsevPetviashvilii equations (a higher dimensional member of the same hierarchy of the KdV equation, see Kadomtsev and Petviashviliĭ, 1970, and Zakharov et al., 1984). We have paid no attention to them in view of the fact that the study of disorder in more than one dimension is still a completely unexplored land.

\subsection{Solitons}

The equations we have listed in the preceding subsection are very different from each other and enter physics due to largely different reasons. So, aside from the obvious fact that all of them are nonlinear, what is their common characteristic? The answer is simple: all those equations share the property that they support the propagation of "solitary waves". The concept of solitary wave was first used by Scott Russell (1844), and its meaning seems rather evident. However, nowadays it has been applied to a lot of objects, and there is no suitable definition to encompass them all. As an illustration, consider the one of Scott et al. (1973): they started with the "travelling wave", that is a solution of a wave equation which depends upon $x$ and $t$ only through $\xi \equiv x-v t$, where $v$ is a fixed constant; then, they picked out solitary waves, which are travelling waves whose transition from one constant asymptotic state as $\xi \rightarrow-\infty$ to another (not necessarily the same) at $\xi \rightarrow \infty$ is essentially localized in $\xi$ (such a transition usually brings along 
with it the localization of the total energy and momentum contained in the system in the same region). This precise definition does not include objects as ubiquitous as sG "breathers" or NLS solitons, for instance. So, we will cling to a rather loose working definition and consider in the following solitary waves those "which propagate without change of form and have some localized shape" (Dodd et al., 1982).

Soliton is a more restrictive concept, and it was introduced by Zabusky and Kruskal (1965). They were performing numerical experiments on the KdV equation (6) and discovered that an initial periodic pulse evolved into a train of solitary waves of steady amplitude that were able to pass through each other without changing their shape or speed, suffering only a small shift in their phase. They termed these special solitary waves "solitons". This finding was really surprising at that time, due to the general belief that if nonlinear excitations collide, nonlinearity would destroy them instead of allowing their crossing. Since then, the word soliton has been more or less reserved to solitary waves of "exactly integrable systems", but it is not rare to find it used to refer to waves of non-integrable equations that are sufficiently localized (exhibiting particle-like behavior, which is the reason for the name "soliton") or show remarkable stability properties. We could even say that in a certain sense, such non-integrable equations are close to some related integrable system.

Not all the equations in our list belong to the class of exactly integrable systems, i.e., the ones for which the general solution of the Cauchy problem can be found. For instance, the $s G, \mathrm{KdV}$ and NLS equation are integrable, while the $\phi^{4}$ or DsG are not. The deep roots for an equation to be integrable or not are still a very intriguing question, and we will not comment on this controversial point here. Let us just say that it is generally agreed that an integrable system has the following properties: it possesses an infinite number of conserved quantities, $\mathrm{N}$-soliton solutions for arbitrary $N$, Backlünd transformations that allow to construct new solutions from already known ones, and exhibit elastic collisions between solitons, the phase shift of a soliton after colliding with a group of another solitons being the sum of the individual phase shifts. All these properties go together, and they constitute a kind of definition for integrable systems (it must be pointed out that there is no commonly accepted definition of "integrability"). However, nonintegrable systems can still have localized solutions, that is to say, solitary waves. This is the case of the $\phi^{4}$ equation or the Maxwell-Bloch system, for instance (Dodd et al., 1982).

On the other hand, solitons are not always identical objects for all integrable systems; indeed, solitons can be divided into three general types: dynamical solitons, topological solitons and envelope solitons. This classification is by no means arbitrary, nor is it useless; rather well, it will hopefully become clear at the end of the review that the three kinds of solitons behave in their own fashion when disorder disturbs their propagations. Let us make a brief description of these classes, starting from the first one, dynamical solitons. Though, in principle, 
all solitons are more stable against perturbations than linear waves, dynamical solitons are the least robust of them, and many times they show quasi-linear behavior (they are amortiguated by dissipation up to their total extinction, for instance). Typical examples are $\mathrm{KdV}$ solitons, whose corresponding one-soliton expression is

$$
\phi_{\kappa}(x, t)=-2 \kappa^{2} \cosh ^{-2}\left[\kappa\left(x-4 \kappa^{2} t-x_{0}\right)\right],
$$

where $4 \kappa^{2} \equiv v$ is the soliton velocity and $x_{0}$ is the position of its centre at $t=0$. See that they are actually a family of solutions, depending on the real parameter $\kappa$, and once $\kappa$ is fixed, all other characteristics of the soliton get fixed too, even its amplitude. A non-integrable equation that possesses dynamical solitons is, e.g., the Maxwell-Bloch system (see Dodd et al., 1982).

Topological solitons depend also on one parameter only, but they are a little more complicated. They can exist only in nonlinear systems with two or more equivalent (or at least similar) ground states; in this case, topological solitons, also called "kinks", connect a part of the system in one ground state to other in a different ground state. The archetype are sG kinks; simple inspection allows to say that constants of the form $\phi=2 k \pi, k=0, \pm 1, \pm 2, \ldots$ are solutions of the $\mathrm{sG}$ equation, Eq. (2). The kink solution are then links between spatial intervals at which $\phi=2 k \pi$, of the form

$$
\phi_{v}(x, t)=4 \arctan \left[\exp \frac{ \pm(x-v t)}{\sqrt{1-v^{2}}}\right]
$$

if the sign in the exponential is a + , we have a kink (going from 0 at $x \rightarrow-\infty$ to $2 \pi$ at $x \rightarrow \infty$ ), and in the opposite case we have an antikink (going from $2 \pi$ to 0 ). Kinks and antikinks are again able to pass through each other without losing any energy. It turns out from Eq. (2) that it is invariant under Lorentz transformations, and so its soliton has been proposed as a model for extended, relativistic particles (Perring and Skyrme, 1962; it must be remembered that in this work the soliton nature of (14) was found via numerical simulations); this is of course connected with the limitation of the speed values, that must verify $v<1$. The topological character provides these solitons with a structure that forbids, among other things, that dissipation wipe them away, being only able to stop their motion. There also topological kinks in non-integrable system like the $\phi^{4}$ equation, and, in general, in all NKG models (Rice et al., 1976).

To finish, let us devote some words to envelope solitons, whose nature is quite different from the other two types. They are two parameterical solitary waves, and their most well-known example is the one-soliton solution of the NLS equation (5): 


$$
\phi_{\xi \rho}(x, t)=2 i \rho \frac{\exp \left[-2 i \xi x-2 i\left(\xi^{2}-\rho^{2}\right) t-\phi_{0}\right]}{\cosh \left[2 \rho\left(x+2 \xi t-x_{0}\right)\right]} .
$$

Here, $-2 \xi$ is the soliton speed, $2 \rho$ its amplitude, $x_{0}$ its initial position and $\phi_{0}$ its initial phase. As it can be seen from (15), the soliton is the envelope (and after this property is named) of a wave packet, coming from the exponential in the numerator. There is another example of this type of soliton: the sG breather, which has heen the subject of many works too; we will come back to it below. The properties of envelope solitons are rather complicated, and their study is complicated by the new degree of freedom, giving rise to an extreme richness of behaviors, that can go from annihilation by friction to stability and undisturbed propagation under rather strong perturbations. As before, we conclude their survey with an example of non-integrable envelope solitons: small amplitude excitations in NKG equations, like those of the $\phi^{4}$ or DsG (Newell, 1985; Remoissenet, 1986).

\subsection{Models of disorder}

So far, we have been presented a number of nonlinear models, and made some remarks on their properties, their applications, and related matter. Now it is time to address the other issue we posed above: how can we model the influence of disorder on these systems? To answer this question, it is not necessary to specify if we are working on a linear or on a nonlinear model. As we already said, the physicists' interest on disorder in nonlinear disordered systems is quite recent. However, they have been studied in the framework of linear problems since some decades ago (let us quote here the name of Lifshitz, probably the most important contributor to this branch of theoretical physics, starting as early as 1937; see Gredeskul and Pastur, 1985), and further developments concerned mainly in the contexts of optics and condensed matter physics (see the reviews by Barabanenkov et al., 1970 and Elliot et al., 1974, respectively, and references therein; it is also worth taking a look at the book by Chernov, 1960). Probably the most important discovery related to linear disordered problems was that of Anderson, namely the localization of quantum wave functions in a random lattice (Anderson, 1958, see below; see also the reviews by Thouless, 1974, and Economou, 1990). Since the middle of this century, there has been a large amount of research on this topic, and a lot of knowledge has been achieved concerning disorder in linear systems. The situation is not so optimistic with respect to nonlinear media, and a lot of things remain to be done.

Either if one deals with linear media or with nonlinear media, several forms of disorder can be considered in every context. The basic and quite useful reference when talking about models of disorder is the book by Ziman (1979). Its first line reads:" 'Disorder' is not mere chaos; it implies defective order. To think 
about a disordered state we must have in mind an ideal of order from which it falls short". In other words, the effect of disorder is always to break some symmetry, forbidding the use of many powerful mathematical tools which rely upon the assumption of perfect order. Ziman (1979) classifies disorder into three types: substitutional, cellular and topological, going from the weakest to the strongest type; on the other hand, they can happen both either in lattice models or in their corresponding continuum representations. Let us briefly define these kinds of disorder. Substitutional disorder occurs when the system under consideration is a perfect lattice, but the components of the system that are associated to each site are not always the same. Think, for instance, of a crystal formed by a single element in which some impurities appear at different places. In a continuous model, this can take place as a variation of mass density in certain zones. Obviously, this form of disorder can be more or less complex depending on the number of unequivalent components that are introduced in the model, their concentration, etc. The most complex substitutional disorder is in fact cellular disorder; this is nothing but an array of cells, topologically ordered, but showing specific features in the composition or properties of each one of them. The best example of this type is ice disorder (Ziman, 1979): in ice, the crystal structure is supported by hydrogen bonds between oxygen atoms, and the molecules in each site are quite similar to those of free water, but they are not exactly equal to each other. The systems of this kind can show short-range order, if the neighboring sites show similar characteristics but this similarity (correlation) decays in a few lattice spacings, or long-range order if it is not so and large parts of the system exhibit approximately the same behavior. Finally, if even the concept of a lattice does not apply, we are in the presence of topological disorder, and, obviously, this is the case for gases, amorphous materials, etc.; in this context, liquids can be classified into a not so strong topological disorder, that can be called dislocation disorder. A detailed description of all the possible types of disorder is clearly out of the scope of this review, and one can find good ones in the already quoted book by Ziman (1979) and in the monography by Lifshitz, Gredeskul and Pastur (Lifshitz et al., 1988), to mention only two. The above general classification is enough for our purposes, provided that we have always in mind the following three remarks. First, each physical system belongs to its own subclass with its particular features (we can say of certain compound that it is a substitutional disordered alloy of a metal with certain percentage of only another one metal uniformly distributed, for instance). Second, disorder may be static and dynamic (going on with the same example, the foregoing metal atoms in the alloy can be fixed or they can diffuse through the host). It is easy to understand that the properties of propagation of excitations, linear or nonlinear, in a system will depend on whether the disorder they find along its trajectory is fixed or change with time. Thus, barriers can disappear, allowing propagation, or can appear, pinning waves; fluctuations can originate radiation and slow waves can cause instabillties, and so on. This new ingredient, time dependence, can 
originate disorder (dynamical disorder, following the terminology by HernándezGarcía, 1989, and Hernández-García et al., 1989a, 1989b, and references therein) in systems otherwise perfect: as an example, think of thermal fluctuations of atoms around their equilibrium positions, always present in any system with positive temperature, and the influence those fluctuations have on waves propagating in the system. Third, as a last remark, to study disorder in a model is not the same as to study model of disordered system. Let us clarify this point with an example: think of the $\mathrm{KdV}$ equation, Eq. (6), as a law for evolution of long dispersive waves, and suppose we want to compute the effect of the inhomogeneities of the medium on soliton propagation. Then, one is tempted to pose the following problem:

$$
\phi_{t}+\alpha \phi \phi_{x}+\phi_{x x x}=f(x) \phi
$$

$f(x)$ being some function (deterministic or random) that should represent the changes of certain parameters depending on the space variable. This naive representation is not what is intended to be, and the reason is at a deeper level: simply, physics has not been properly taken into account in deriving Eq. (16). Equation (6) describes long waves in a moving reference frame of velocity $V$, that must be fixed to select waves propagating in only one direction, as we said above, and its primitive form is

$$
\phi_{t}+\alpha[\phi-V] \phi_{x}+\phi_{x x x}=f(x) \phi ;
$$

if we now change variables, $x \rightarrow x^{\prime}+V t^{\prime}, t \rightarrow t^{\prime}$, we find

$$
\phi_{t^{\prime}}+\alpha \phi \phi_{x^{\prime}}+\phi_{x^{\prime} x^{\prime} x^{\prime}}=f\left(x^{\prime}+V t^{\prime}\right) \phi
$$

evidently different from (16). In (18), spatial disorder is now correctly included. But this must not be interpreted as if there were no reasons to study Eq. (16). This is out of the question. Equation (16) is as interesting as any other. The very point is that it is a simple example of a problem concerned with disorder in the $\mathrm{KdV}$ model, and it is relevant, e.g., to stability properties of solitons; on the other hand, Eq. (18) is an example of a problem that deals with a system modeled, when unperturbed, by a $\mathrm{KdV}$ equation, and then it constitutes a model of a real disordered system.

The preceding comments on what disorder is lead to the next point, namely the mathematical representation of it. It seems natural to have the choice of several kinds of random variables and stochastic processes as the most suitable form to model disorder. Indeed, randomness is often used as a synonym of disorder. The way to introduce it in each model depends on what kind of problem we are going to address. Therefore, we can consider a lattice of equal impurities (e.g., with identical potentials) randomly arranged in space, or a 
perfect array of impurities of different kinds and intensities, or both; we can allow these impurities to move in the unperturbed system or to change their nature in time; we can allow them to be discrete (and consequently use a set of random variables) or to encompass a whole zone of the model (what corresponds to a stochastic process); we can consider a field that is the same along the entire system but fluctuates in time, again discretely or continuously; the correlation between random variables or of the chosen stochastic process can have long range or be a delta function depending on their real counterparts, etc. The list would be never-ending, and it is enough that these few examples (and the ones that will appear in Secs. 3 and 4) show the role of stochastic variables in hiding our ignorance on certain physical problem.

Before closing this subsection, a few words are necessary concerning two remaining disorder models. The first one refers to a weaker form of topological disorder, that of quasicrystals, which are structures in which there is no strict order but the components are arranged following certain deterministic, reproducible rule, like that of Fibonacci sequence, for instance (see Steinhardt and Ostlund, 1987). Still at its birth, the study of wave or particle propagation through quasicrystalline structures has already yielded very interesting findings, like the complex (fractal, in fact) structure of the spectra of such structure (Kohmoto et al., 1983; Ostlund et al., 1983). More well established is the interest on fractals, that are perhaps the most novel model of disorder. The concept of fractal objects was introduced by Mandelbrot in 1975 (Mandelbrot, 1975) and it reached maturity in his very famous book on fractal geometry (Mandelbrot, 1982). Fractals are very complex systems or objects, which show non-trivial structure in all (or a wide range of) scales, and, moreover, they seem to appear in an ubiquitous manner in nature (Mandelbrot, 1982). Along this decade, they are entering the field of Condensed Matter Physics from several viewpoints, and, though deterministic fractals (i.e., those which are constructed following a rule) are not disordered in a strict sense, they, as well as random fractals, are more and more employed to model complex disordered structures, resulting from certain growth processes (Vicsek, 1989) for instance, or from many other different phenomena (Feder, 1988). In connection with this aspect of disorder, the basic references that the interested reader should look in order to get an idea of the use of fractals as disorder models are the Proceeding edited by Pynn and Skjeltorp (1985), Stanley and Ostrowsky (1985), Pietronero and Tossatti (1986), Pynn and Riste (1987), Pietronero (1989), and Aharony and Feder (1989). Fractals can give rise to very new and striking phenomena, like superlocalization (Levy and Souillard, 1987) or anomalous diffusion (Gefen et al., 1983; see also the review by Havlin and Ben-Avraham, 1987). Besides, the recent introduction of multifractals (see the review article by Stanley and Meakin, 1988, and references therein) is opening a lot of new fields to research and providing new tools to classify objects and process. We are not going to talk about quasicrystals and fractals in the rest of this the paper, because, to our knowledge, nonlinear 
problems have not been studied when perturbed by or happening in such substrates, or potentials, or whatever; we just pointed them out as the first open question we mention, a very fascinating subject that will certainly be important in the near future.

\section{Techniques}

We will split this section into two parts, devoted separately to analytical and numerical techniques. However, as we have already remarked in the introduction, in every nonlinear problem it is crucial to do a judicious use of both, because not only do they provide complementary information, but also serve as a checking of results. This checking is always necessary: from the analytical point of view, all of the results are usually in one or another perturbative, and hence valid only in a certain range to be determined a posterior by means of simulations; from the numerical point of view, discrete systems may not show exactly the same dynamics as their underlying continuous versions, and one has to filter out the spurious numerical outcomes checking analytically known properties. We will always have in mind this collaboration in what follows. On the other hand, we do not provide a total description of each technique but only their main lines and further references where a thorough description can be found.

\subsection{Analytical techniques}

It is well known that all the possible nonlinear partial differential equations that can be imagined are non-integrable, except for a very few of them. Even more: when these last ones are perturbed, the probability that they still remain integrable is negligible. It is non zero: sometimes, it is possible to find a change of variables such that the perturbed equation is cast into the form of an integrable one. Let us show some examples. The first one is again related to a perturbed $\mathrm{KdV}$ equation, similar to that we already picked up for the same purpose in the previous section. We have chosen it from the works by Wadati; he studied analytically (Wadati, 1983) and numerically (Wadati and Akutsu, 1984) the Korteweg-de Vries equation under thermal fluctuations, including the so-called additive noise

$$
\phi_{t}+\alpha \phi \phi_{x}+\phi_{x x x}=\xi(t)
$$

where $\xi(t)$ is a gaussian white noise, and showed that by putting

$$
\psi(x, t) \equiv \phi(x, t)-\int_{0}^{t} d t^{\prime} \xi\left(t^{\prime}\right)
$$


the usual Korteweg-de Vries equation, Eq. (6), is recovered. Subsequently it can be solved and the solutions averaged to get their statistical characteristics (by the way, he was able to prove that solitons were wiped away by noise, that transformed them into Gaussian wave packets whose amplitude was gradually diminishing). Wadati (1990) has applied the same procedure to solve a Boussinesq stochastic equation derived in the continuum limit for a Toda lattice with random masses. In fact, it is possible to use the same change of variable for a wider class of equations that includes several NLS problems (Bass et al., 1988). The second example come from perturbed sG systems: it has been shown (Khikmatov, 1982) that the equation

$$
\phi_{\chi \tau}+[1+\xi(\tau)] \sin \phi=0,
$$

which is nothing but the sine-Gordon equation in light cone coordinates $2 \chi=x-v t, 2 \tau=x+v t$, can be solved with the help of the transformation

$$
\zeta \equiv k \chi-\frac{1}{k}\left[\tau-\frac{1}{2} \int_{0}^{2 \tau} d t^{\prime} \xi\left(t^{\prime}\right)\right]
$$

$k$ being a constant parameter, that allows to cast Eq. (21) into sG form, Eq. (2) for the variables $\zeta$ and $\tau$. However, the question arises about the meaning of a noise that depends on the coordinate $\tau$. Furthermore, even for a equation with more evident physical meaning as (19), the following, more severe drawback must be had in mind: after changing variables and getting the usual $\mathrm{KdV}$ equation, one knows its exact solution, but it will depend on a stochastic position arising from the change itself. If one tries to see what happens to a soliton, and then chooses a soliton solution for such equation and averages to obtain mean values, he will be averaging over a set of solitons with random coordinate origins, cf. Eq. (20), and it is not so clear what will the result of such averages mean physically. Think that usually the effect of these random perturbations on a soliton is not only to induce its motion; instead, it generates additional radiation, and radiation is not being included in the ensemble for averages because we are imposing soliton shapes. So, we get the unpleasant conclusion that, very often, the few disordered problems that are exactly solvable are rather academic, and their physical applications are not very clear. Yet, there is one advantage of these computations we should stress: by their means it may be possible some times to predict general laws for the system that can be used to check numerical simulations of stochastic partial differential equations, a task that it is never easy.

Therefore, we must be realistic and forget about solving our particular problems exactly, and try to get some approximate results that can still be useful. The first idea in this direction is the following: when dealing with nonlinear, partial differential equations, the problem is that of a system with an infinite number of degrees of freedom. Might it be possible to "remove" most of these 
degrees of freedom, and keep only a few of them? If such a procedure exists, then the problem is reduced to that of a set of ordinary differential equations, which is far easier to study and to get physical conclusions. The answer to this question is the collective coordinate method. To be strict, there is no one collective coordinate method, but some of them that share the same fundamental idea: solitons behave like particles. This idea was proved by Fogel et al. $(1976,1977)$; (see also Bishop et al., 1980) for sG solitons, although it applies in general; since then, collective coordinates have been widely employed. The central point is to choose one or several variables, like the center, the speed, the width, the frequency of an oscillatory degree of freedom (like in $\phi^{4}$ kinks or NLS solitons), etc., for the soliton, and neglect any other effect of perturbations, like radiation emission. Subsequently, by one of a variety of means, one derives equations for the evolution of the selected variables that are amenable to powerful analytical approaches. The final stage is to evaluate the neglected contributions to see if they were actually small. Incidentally, and as a first illustration, the three equations of the Lorenz system (Lorenz, 1963) were derived by a similar reduction of the number of degrees of freedom from the Navier-Stokes equation. In spite of its apparent drastic simplification, this approach is very useful as an order zero study of perturbed problems, and, even more, for many practical purposes it is not necessary to go beyond it.

Once the set of coordinates is chosen, equivalently, once some ansatz for the solution of the perturbed equation is fixed, with a number of parameters depending on time being the collective coordinates, there are two simple ways to derive equations of motion for them. The first one is to use the conserved quantities of the unperturbed system (see examples in McLaughlin and Scott, 1978; Rice and Mele, 1980; Bergmann et al., 1983, or Pascual and Vázquez, 1985) and it consists of computing their variation under the influence of the perturbation in two ways: inserting the ansatz in their definition and taking time derivatives, or use the perturbed equation of motion to deduce this time derivative for a general solution and after insert the ansatz. Equating the so obtained expression leads to the desired evolution laws. The other procedure is only valid for Hamiltonian systems; the ansatz is introduced in the Hamiltonian, the value of this magnitude computed, and subsequently Hamilton equations for the parameters are obtained. Of course, it is also possible to use the Lagrangian formalism. It must be added that recently another approach (projection-operator approach: see Boesch et al., 1988, and references therein) has been developed in the framework of NKG equations, based on Dirac's treatment for constrained Hamiltonian systems. As a last remark, it is necessary to be careful about the equations for the collective coordinates, because the possibility exists that they turn out to be ill posed and give spurious divergences due to improper choices of coordinates (Caputo and Flytzanis, 1990; see also Flesch, 1987, which contains 
a detailed analysis of the collective coordinates for NKG problems.) Examples of this formalism can be found in Nozaki (1982), Malomed (1987), or Campbell et al. (1991) to quote just some of them.

A second way to study perturbed nonlinear systems is to try perturbative expansions, following the customary procedure. The solution to the perturbed equation is written as a series in some small parameter, to which the perturbation is proportional. The zero contribution to this series is a solution of the unperturbed equation. After substitution in the perturbed system and removing this zero order, one is left with an in principle infinite sequence of equations, each one containing terms coming from the lower orders. This is usually cut at first order supposing the expansion parameter is small enough; on the other hand, higher orders become cumbersome in general. The first order approximation of this so-called direct perturbation expansion is often termed Born approximation. The first and well-known example of such a technique is that of Fogel et al. (1976, 1977), who employed it to study the sG system. Notice that the first order equation is also nonlinear and a further assumption is made, namely that corrections are small. Then one can linearize that equation and obtain the nonlinear radiation modes that describe the excitations about the starting solution. Examples of this technique, that can be applied to any equation no matter it is integrable or not, can be found in Bass et al. (1988) and references therein. An improvement of these kind of perturbative expansions was proposed by McLaughlin and Scott (1978), and is simply to introduce some free, time-dependent parameters in the order zero term, i.e., in the exact solution of the unperturbed problem. The equations for their evolution are then obtained naturally as conditions for the absence of secular terms. It is quite remarkable that if one chooses an ansatz for the extra variables among those of the collective coordinates type, the equations of motion are the same, no matter whether they are derived by the above described means or from this secular perturbative expansion. Sometimes free parameters are allowed to evolve in a different time scale than the radiation modes, and then one can take advantage of the multiscale method (Morse and Feschbach, 1953) to deal with the equations (see, e.g., Konotop et al., 1990) as an illustration.

This direct perturbation method and its variants apply to any nonlinear system, independently of its integrability. However, if the unperturbed system is integrable, one can obtain more benefit by means of the perturbation theory based on IST. There are two main branches of IST perturbative theories. The first one is related to direct perturbation theory: when one is solving for the radiation contribution, a basis of eigenfunctions of the linearized problem is needed. Then, if the IST solves the unperturbed equation, it is possible to use it to obtain this basis (Keener and McLaughlin, 1977a, 1977b; McLaughlin and Scott, 1978; Mineev and Shmidt, 1980) computing a Green function (see Flesch and Trullinger, 1987, for a recent derivation of Green functions for NKG problems without IST). The other branch could be considered as an ab initio IST perturbation theory, 
introduced by Kaup $(1976,1977)$ and Karpman and Maslov (Karpman, 1977; Karpman and Maslov, 1977, 1978). We will not go into the detail of this theory, nowadays a well established one, but refer the reader to the recent, thorough review by Kivshar and Malomed (1989) and references therein; let us say only that, as the IST can be considered as a generalized transformation to action-angle variables (the so-called scattering data), that evolve in time in a trivial way, the IST perturbation theory is based on the knowledge of the evolution in time of these quantities under the effect of perturbations. Again, this is found as an expansion on powers of some small parameter, and the equations for the collective coordinates are once more recovered in this formalism starting from an order zero term with time dependent parameters.

This section would not show the main viewpoints if we do not make some comments on stochastic techniques (see Kaup and Osman, 1986, where a singular perturbation theory specially suitable for stochastic problems is developed). In principle, all the already mentioned techniques are valid for deterministic or random perturbations. The problem in this last case is obtaining the quantities one is interested in, like mean positions, mean emitted energy, etc.; in general, one will be looking for certain averages. This is not at all trivial even if one is able to apply any perturbative technique. Often, it can be useful to start from a more probabilistic departure point, namely trying to find probability distributions for the variables of interest. Difficulties arise then from the infinite number of degrees of freedom: if one tries to write a Fokker-Planck equation (van Kampen, 1981; Gardiner, 1983; Risken, 1984) that describes the evolution in time of the probability density, the result is a functional equation that is practically useless. A reduction in the number of degrees of freedom is in order to proceed through this method, and it can be achieved either by a previous collective coordinates treatment (Base et al., 1985), or restricting the desired knowledge of radiation properties to a total emitted energy (Kivshar et al., 1986), and so on (see Bass et al., 1988, for a review on this problem). If the system under consideration possess a Hamiltonian structure, there exist also the possibility of using it to derive equations for the energy, momentum and other magnitudes of the system (Parrondo et al., 1990; their procedure is even valid for colored noises with not too large correlation times).

\subsection{Numerical techniques}

The basic methods to solve numerically partial difference schemes, finite element schemes, Monte Carlo procedures, spectral methods and variational methods (Press et al., 1989). Among them, the most often used techniques in nonlinear physics are finite difference and spectral ones. Historically, aside from the work of Fermi, Pasta and Ulam (Fermi, 1955), which was already discrete, i.e., defined on a lattice of spatial points, the first numerical works are due to Perring and Skyrme (1962) for the sG equation and to Zabusky and Kruskal (1965) for the 
KdV system, both of them using finite difference schemes. The idea of finite difference schemes is to substitute the spatial coordinate by a grid or lattice of points, or in other words, to change from the variable $\phi(x, t)$ defined on the whole (or a part of) the $x$ axis, to the set of variables $\phi_{n}(t) \equiv \phi(n \Delta x, t)$; in this fashion, the partial differencial equation is transformed into a finite number of ordinary differential equation. Further discretization of the time variable leads to the scheme for integrating the original system. Usually, the forms of discretizing the time variable are more than those of the spatial coordinate, this being due to the fact that there are a lot of different procedures to deal with ordinary differential equations (see, for instance, Press et al., 1989). At first, the schemes were really simple ones: Euler, leapfrog, or second order Runge-Kutta procedures. However, nowadays, things have changed, with the availability of quite fast machines at reasonable prices and ease of use, and more precise methods are customarily used.

It goes without saying that finite difference schemes should provide numerical results that reproduce the essential analytical features of the underlying continuous equation in order to avoid the numerical chaos (Herbst and Ablowitz, 1989a). These essential features are: conservation of quantities (energy, momentum, mass,...), symmetries (under time inversion, for instance), stability properties of solutions, conservation of phase space volume (for Hamiltonian systems), and so on. For each particular problem one should pay attention to these and similar characteristics and try to reproduce them numerically as accurately as possible; this is always the best criterion to choose a scheme for a numerical simulation. On the other hand, discrete problems carry along with them intrinsic differences with respect to their continuous counterparts, arising as new dispersion relations for quasi-linear, small amplitude waves propagating in the system, or the presence of numerical boundaries due to the finite size of the simulation versus PDE's defined in the whole line. It would be very tedious to enter in depth into these questions; the interested reader should take a look at the papers by Trefethen $(1982,1984)$ and references therein, for instance.

Therefore, attending to their properties, we can classify finite difference schemes into four main categories: conventional (i.e. not showing the special features of the other ones; examples are Runge-Kutta or predictor-corrector methods used to solve the set of ordinary differential equations arising from discretization of space); conservative (that is to say, they conserve discrete analogs of one or some of those of the continuous model); symplectic (those that exhibit canonical behavior, preserving the volume of their discrete phase space in the evolution) and finally variational, that by means of a free parameter allow to obtain conservative, symplectic schemes. All of them are good when used in simulations of a short time interval, the high-order Runge Kutta ones being probably the most accurate. However, when the necessity arises to extend the simulation to longer times, most of them show unphysical chaotic behavior (see, for instance, Herbst and Ablowitz, 1989b, for chaos in NLS schemes, Goedde et 
al., 1990 for the same subject in sG schemes or Jiménez and Vázquez, 1990, for general NKG equations), that has nothing to do with the real solutions of the model. This is actually dangerous in problems involving stochastic variables: if there is any chaotic evolution, one must be sure that it arises due to the equation randomness and not due to the equation discretization, or, otherwise, it will never be known what is the real dynamics of such system. To this respect, only conservative systems appear to be free of this phenomenon, quite likely because the conservation of quantities restricts their evolution in the discrete phase space to non-chaotic regimes. Nevertheless, careless use of any finite difference scheme, even the conservative ones, can lead to spurious effects, like for instance "pinning" of solitons, first discovered by Currie et al. (1977) in the sine-Gordon system (see good descriptions for the $\phi^{4}$ model but of rather general applicability in Combs and Yip, 1983, 1984, or Kunz and Combs, 1985); this phenomenon is nothing but the slowing down of solitons propagating in a discrete lattice due to some induced emission of radiation not present in the continuum model, and takes place when the lattice spacing is not much smaller than the width of typical solitons.

To be specific, we will summarize some schemes and their features for NLS and NKG models. Other models like $\mathrm{KdV}$ are more amenable to analytic work (though of course they have not been forgotten: see Zabusky and Kruskal, 1965, or Dodd et al., 1982, and references therein for numerical schemes to work with the KdV system), or are not so widely applicable as these two are; hence, it is not exaggerated to say that almost all of the known results on numerical analysis of partial differential equations refer to this two (aside of course from diffusion equations, with which we do not deal here). Concerning the NLS equation, a recent survey of results is provided by the paper by Strauss (1989) for nonlinear Schrödinger equations of the form

$$
i \phi_{t}+\Delta \phi+\lambda|\phi|^{p-1} \phi=0, \quad p>1 ;
$$

for this type of equation there are some conserved quantities, like charge, $N=$ $\left.\int d x\right\rfloor\left.\phi\right|^{2}$ (the integral is taken over the whole axis), energy, $E=\int d x \frac{1}{2}\left[|\nabla \phi|^{2}\right.$ $\left.-\frac{\lambda}{p+1}|\phi|^{p+1}\right]$ and the momentum, $P=2 \operatorname{Im} \int d x \phi \phi_{x}^{*}$, that can be the basis for valuable checkings of the numerical integration; if the scheme does not conserve them exactly, their variation should be tracked to ensure that it is not large (a large deviation would be equivalent to an evolution without any physical meaning). This applies when studying perturbations of Eq. (23) in the sense that theses quantities will not be conserved anymore, but their change will follow certain laws given by the corresponding evolution equations. This change should be reflected in the scheme when including the discretization of the perturbing terms. Several methods have been proposed for the numerical simulation of NLS problems (Ablowitz and Ladik, 1976; Delfour et al., 1981; Griffiths et al., 1984; Taha and Ablowitz, 1984; Herbst et al., 1985; Sanz-Serna and Verwer, 1986; 
Guo Ben-Yu, 1986) and among them we will point out the one by Ablowitz and Ladik (1976), already written down (see Eq. (12) above) and solvable via IST, that has been recently compared successfully with other schemes with chaotic features (Herbst and Ablowitz, 1989b), and the one by Delfour et al. (1983), that is conservative. It is worth mentioning also an implicit (i.e., to compute the solution at the point one is interested in, a transcendental equation must be solved, generally by Newton's method) scheme proposed for logarithmic NLS equations (Brito et al., 1988) which can be generalized in an explicit (which means that the corresponding equation is straightforwardly solvable) form for cubic NLS equations (Jimenez, 1990).

Concerning NKG models (1), there are also two quantities that serve as a test for any scheme: energy $E=\int d x\left[\frac{1}{2}\left(\phi_{t}^{2}+\phi_{x}^{2}\right)+V(\phi)\right]$, and momentum, $P=$ $-\int d x \phi_{t} \phi_{x}$. A lot of different schemes has been proposed, but the situation has been recently clarified by a paper by Jiménez and Vázquez (1990). They addresed a question posed in tbe book by Dodd et al. (1982), that stated that a detailed comparison should be between the two main procedures to simulate NKG equations: those of Strauss and Vázquez (1978) and Ablowitz et al. (1979). The comparison is presented in the quoted work including also two other schemes, and it turned out that, while the one of Ablowitz and coworkers was somewhat more accurate for shorter times, all the schemes but the Strauss-Vázquez showed unphysical features and blow-ups for large times or large amplitude initial data, in spite of the fact that it can be analytically shown that the solution to the equation must remain bounded. So, it seems to us that this is up to now the best finite-difference procedure for NKG models. This is more so taking into account its good properties in stochastic problems, for which it is the only scheme that has been tested against exact results (Sánchez and Vázquez, 1990; Sánchez et al., 1991).

Of course, we have to face the questions posed by stochastic problems. It is an unfortunate fact that people working in stochastic processes have focused their work mainly in ordinary differential equations, and subsequently they have contributed very few results to the study of solitons under random perturbations. The most useful reference is the review by Mannella (1989), where some techniques are summarized for systems of ODE's, or, equivalently, discretized PDE's. It is worth noting that from the numerical point of view, there are two questions that should be considered before trying any simulation. The first one is related to the generation of random numbers. Random numbers provided by an algorithm are not random, but pseudo-random. The point is to obtain sequences that are similar to actual random numbers. This is a matter that should be studied carefully. There is no general procedure that is guaranteed to work in any machine: algorithms are machine-dependent, because they are usually based on the finite arithmetic of computers (and hence in their architecture) to simulate random variables. The basic references about this topic are the books by Knuth (1981) and Press et al. (1989). In any case, the user of random number generators 
must be extremely careful, and tests of their temporal correlations (not only binary but some high order correlators) are strongly recommended. There is even one more remark concerning stochastic PDE's, and it is related to stochastic processes. Once a reliable random number generator is available, there is no trouble in dealing with a problem in which, for instance, several impurities are placed at random positions. But if we are interested in an equation which involves a stochastic process, like that of thermal fluctuations, or multiplicative noises, it must be realized that their discretization does not have the same properties as the continuous one. The exact properties of the discrete analogue must be computed in every situation to see that they should be in order to build an actual analog of the desired process. For instance, if we want to simulate a gaussian white noise, say $\xi(t)$ (often required, not only by its own interest but also to construct non-delta correlated processes) with correlation $\left\langle\xi(t) \xi\left(t^{\prime}\right)\right\rangle=$ $D \delta\left(t-t^{\prime}\right)$, we have to use a gaussian random number generator with correlation $\left\langle\xi_{n} \xi_{m}\right\rangle=D(\Delta t)^{-1} \delta_{n m}$ to represent the average effect of noise in the time interval $\Delta t$ (see, e.g., Pascual and Vázquez, 1985; Rodríguez-Plaza and Vázquez, 1988, 1990, or Sánchez and Vázquez, 1991). This requires the noise to be interpreted in the Stratonovič sense (van Kampen, 1981). In this context, we want to stress that, in general, all stochastic problems have some definition subtleties that should be handled with care, and the used procedure and its interpretation should be specified in each work for the sake of reproducibility.

The main advantages of the finite differences methods are their straightforward character, that makes their implementation for each computer an easy task, the amount of available literature on them, and their suitability for a large number of equations; moreover, finite difference methods are the customary choice to implement molecular dynamics codes to study the statistical physics of nonlinear lattices (see Schneider and Stoll, 1978, and references therein). However, they are neither the fastest methods, nor the most accurate. These characteristics are best fulfilled by spectral (also called function approximation methods), particular choices of them being Fourier Transform or finite element methods. The idea of these procedures is to choose a number of functions that verify the boundary conditions of the problem, decompose the unknown solution in terms of these functions with some time dependent coefficients, and, substituting in the PDE, get some evolution equation for these coefficients (an extra condition can be required to this end, see for instance the books by Mitchell and Wait, 1977, or Mitchell and Griffiths, 1980). Up to date, spectral methods have not been massively employed in dealing with problems of nonlinear wave propagation, the finite difference ones being more successful. This can be due to the fact that, in spite of their fast convergence and high local accuracy, they can be in trouble when discontinuities are present and fail to reproduce the essential analytical features of the continuous equation. Discontinuities are a common situation in this subject because of the presence of Dirac delta functions at several 
points simulating impurities and so on, and hence the number of problems treatable by these spectral means reduces drastically.

Let us now turn to a quite interesting (and in principle surprising, because it refers to the most sophisticated analytical tool) topic, the numerical implementation of IST. Of course, the applicability of such techniques is restricted by definition to IST integrable systems. It relies on the studies of nonlinear problems defined on a finite interval, like that of Forest and McLaughlin (1982) for the sine-Gordon problem, but it can be used to deal in an approximate way with equations for which IST can be performed when defined on the whole line. The main purpose of this technique is to monitor the evolution of a perturbed equation looking for the creation or annihilation of solitons due to the perturbation effects. Indeed, it was first used by McLauglin and Overman (1982) to show how a $\mathrm{SG}$ breather decays into radiation as a consequence of dissipation. Later, it has been used by Overman et al. (1986) to study chaos in damped driven sG systems, by Taki et al. (1989) to study NLS systems and by Caputo and Sánchez (1991) to study parametric sG problems (while we were at the final stages of writing this review, a large paper by Flesch et al., 1991, appeared, devoted to the numerical IST for $\mathrm{sG}$ problems). The idea is one lets the equation evolve in time, usually through a reliable finite difference scheme, and inputs the so obtained profiles in the associated linear scattering problem to analyze them in terms of the nonlinear excitations of the unperturbed problem. This is meaningful if after some time the perturbation is switched off, because for later instants the system evolve according to the number and characteristics of the computed solitons and radiation. The drawback of this technique is the large time needed, and the practical impossibility of applying it to stochastic problems due to similar reasons. On the other hand, its advantage is that this is the only way (without extremely long runnings) to ensure that the outcomes of the perturbation under study are really solitons or only some shapes looking like solitons whith non-solitonic properties.

Finally, although we are reviewing numerical methods, we want to include here some notes on other techniques to simulate soliton-bearing equations: analog computers. There are two main kinds of analog computers. The first are mechanical analogs, real apparatus designed on purpose to verify certain equation. As an illustration, consider the first one, as far as we know, proposed by Scott (1969) for the sG equation. It consisted of a series of pendula connected by steel springs and supported horizontally on a taut length of piano wire. The variable is the angle rotated by each pendulum. It was improved by Nakajima $e t$ al. (1974) to introduce dissipation (a viscous fluid, in fact) and variable masses for the pendula, and another one was employed to study a perturbed sG equation by Cirillo et al. (1981). The other type on analog computers, and maybe the most versatile, are electrical networks, that can be readily modified to take into account 
various perturbing factors. A long, updated list of references, as well as descriptions of the most important ones, can be found in Remoissenet (1989, 1990a, 1990b).

As a closing remark, we would like to quote the following words of the book by Dodd et al. (1982), that still remain fully valid: "As a tool for the study differential equations, numerical analysis offers a confusing variety of techniques for specific equations, the question of the 'best' numerical method is an extremely complicated one. Even a partial answer to this question depends on many factors: for example, the final accuracy required for a specific range of the independent variables, the limitations of time and storage space, and machine word length. Equally important is the amount of time and effort available for the development of the appropriate software. Most computational physicists or applied mathematicians are content to derive one reasonably efficient method and few detailed comparisons are made with other methods. For this reason no outstanding claims are made for the methods described below (above, in our case) except in the cases where meaningful tests have been carried out." The same applies to what we have said; unfortunately, the situation is not more clearer nowadays, nine years later, mainly due to the lack of detailed tests as well as to the fact that, generally speaking, research papers do not provide enough information on their numerical procedures. Anyway, we hope the survey we present here is enough to understand the current state of numerical studies of solitons.

\section{Nonlinear Klein-Gordon Models}

In this section we will concern ourselves with two essentially different nonlinear Klein-Gordon models: the sine-Gordon one, given by Eq. (2), and the $\phi^{4}$ one Eq. (3). The root difference between them is that the former is integrable and the second is not. As we already mentioned in the introduction, this short sentence implies a large number of consequences, about which we will comment below. It makes sense then study both of them separately and we do so in the next two subsections.

\subsection{Sine-Gordon model}

\subsubsection{Generalities}

The study of NKG models can be started from several departure points. Often, the departure point is the Lagrangian formallsm of classical field theory. This has the advantage that allows to present the equation in brief, with respect to its mathematical properties, treating them in a very standard fashion (Rubinstein, 1970; Rajaraman, 1975). Thus, let us recall that all NKG models can be derived from the following Lagrangian: 


$$
\mathscr{L}=\int_{-\infty}^{\infty} d x\left[\frac{1}{2}\left(\phi_{t}^{2}+\phi_{x}^{2}\right)-V(\phi)\right]
$$

It is enough to introduce the appropriate $V(\phi)$ to obtain the desired equation. For the sine-Gordon case, this potential is $V(\phi)=1-\cos \phi$. Notice that the expression (24) is in dimensionless form, which can always be achieved from the raw physical one by appropriate changes of variables (see Eilenberger, 1981, or Dodd et al., 1982, for instance); then, we will always refer to Eq. (24) without loss of generality. Equation (2) follows as the evolution law for the field $\phi$ inmediately from (24) through Euler-Lagrange formalism.

The main property of the $\mathrm{sG}$ equation is that it is exactly integrable (see, for example, Zakharov et al., 1984, or Eilenberger, 1981, for a survey of the IST formulation of the problem and related properties). Among the infinite number of conserved quantities implied by this integrability, we have already mentioned two of them in the previous section, namely the energy and the momentum. There is another important one, the so called topological charge

$$
\frac{1}{2 \pi} \int_{-\infty}^{\infty} d x \phi_{x}=\frac{1}{2 \pi}[\phi(\infty, t)-\phi(-\infty, t)]=N
$$

$N$ being an integer. This quantity is specifically responsible for the stability of the sG kinks, the one-soliton solution (14) that we presented in the section to the types of solitons. There we also mentioned another excitation that occurs in the sG model, the breather (a rather extended description of breathers can be found, e.g., in McLaughlin and Scott, 1978), whose expression is

$$
\phi_{b \tau}(x, t) \equiv 4 \arctan ^{-1}\left[\tan \mu \frac{\sin \left[\gamma(t-v x)+x_{0}\right] \cos \mu}{\cosh \left\{\gamma\left[x-v\left(t-t_{0}\right)\right] \sin \mu\right\}}\right] .
$$

Breathers are oscillatory states (they are named after those oscillations, that remind something like breathing) of frequency $\cos \mu$ and with null topological charge, that can be seen as a bound state of two kinks. So, the general solution of the sG equation (2) for an arbitrary initial condition will consist of a certain number of kinks (and antikinks), another possibly different number of breathers, and radiation which consists of small amplitude nonlinear, periodic excitations, quite similar to linear ones, of wave number equal or greater than 1 .

The sG equation arises in many different contexts, either in the continuous form, Eq. (2), or in its discrete version. We find it useful to present the corresponding discrete Hamiltonian, which is

$$
\mathscr{K}=\sum_{i=1}^{N}\left[\frac{1}{2}\left(\frac{d \phi_{i}}{d t}\right)^{2}+\frac{1}{2}\left(\phi_{i}-\phi_{i+1}\right)^{2}+\left(1-\cos \phi_{i}\right)\right] .
$$


In applications, the sG system is often written in this way, to take into account the discreteness of the considered model. Nevertheless, if the model is not too discrete, i.e., the lattice spacing is much less than the kink width (the natural length scale) then the continuous sG equation yield quite approximately the same results as this discrete one.

Aside from the fact that Eq. (2) is related to Josephson junction electrodynamics (Barone and Paternó, 1982, and references therein), it appears in a lot of subjects, like motion of dislocations in crystals (Frenkel and Kontorowa, 1939; Nabarro, 1967), domain walls in quasi-one dimensional ferromagnets (Enz, 1964; Mikeska, 1978; Kjems and Steiner; 1978) or ferroelectrics (Pouget and Maugin, 1984, 1985a, 1985b), electromagnetic waves in semiconductor superlattices (Bass and Tetertov, 1985), charge density waves (Lee et al., 1974; Rice et al., 1976; Grüner and Zettl, 1985), waves in liquid helium (Maki, 1978; Kitchenside et al., 1978), etc. (see Dodd et al., 1982, or Kivshar and Malomed, 1989 for more references on applications). This list justifies clearly the importance of studying the $\mathrm{sG}$ equation as well as the influence of perturbations on it.

\subsubsection{Point-like impurities and related topics}

To our knowledge, the first study of the influence of disorder, or, to be specific, impurities, on sine-Gordon systems was done by Fogel et al., in two famous papers (1976, 1977 nowadays). They considered the sine-Gordon equation plus different perturbative terms: driving external forces and damping, a model impurity, and spatial inhomogeneities in the coefficient of the nonlinear term. Their way to proceed was through a direct perturbation expansion of the kind we mentioned in Sec. 2. We give some detail on their calculations in view that the description they present of the perturbed kink in terms of a decomposition in different modes has become a standard procedure and terminology, and applies to all NKG systems. In brief, what they did was to suppose that the effect of perturbations on solitons would be the appearance of small oscillations around their unperturbed shape: that amounts to choose an ansatz of the form $\phi(x, t)=\phi_{v}(x, t)+\psi(x, t), \phi_{v}$ being the unperturbed kink given in Eq. (14), and $\psi(x, t)$ being the small correction we are looking for. Subsequently, the ansatz is inserted into the $\mathrm{sG}$ equation; by means of the assumption of small deviations from the kink shape, cubic and higher order terms in $\psi$ are neglected and, after Lorentz transforming to the kink rest frame, one is left with

$$
\psi_{t t}-\psi_{x x}+\left(1-2 \operatorname{sech}^{2} x\right) \psi=0
$$

The next step is to assume harmonic time dependence for $\psi$, i.e., $\psi=f(x) e^{-i w t}$; we then arrive at 


$$
-\frac{d^{2} f(x)}{d x^{2}}+\left(1-2 \operatorname{sech}^{2} x\right) f(x)=\omega^{2} f(x),
$$

which is nothing but a Schrodinger equation with a Hylleraas potential (Rubinstein, 1970), whose solutions are one bound state with $\omega=0$, and a continuum of solutions with $\omega=1+k^{2}, k$ being a real wave number. The first one is customarily called the Goldstone mode, and it can be seen that if it is added to a soliton profile, the result is the same soliton translated in space, so it is also termed the translation mode (see details, e.g., in Fogel et al., 1977, or in Flesch and Trullinger, 1987). The continuum modes are radiation-like ones, and in fact resemble the linear waves of Klein-Gordon equations except for a disturbance localized in the vicinity of the soliton. The importance of these modes is that, as they are solutions of a Schrödinger eigenvalue problem, they form an orthonormal basis of the space of functions of $x$, which allows to decompose the excitation profile $\psi(x, t)$ and compute separately each mode contribution. This is the basis of the computations of Fogel et al. Their technique is simply to insert an ansatz as that above in the perturbed equation, and linearize it; after that, they solve it by writing the correction in terms of modes followed by Fourier transformation in time.

The perturbations they study that are concerned with disorder are two: first, an impurity modeled by a term added of the form $\lambda \phi_{x}\left[\theta\left(x-x_{0}\right)-\theta\left(x+x_{0}\right)\right]$, which gives rise to the following perturbed $\mathrm{sG}$ equation,

$$
\phi_{l t}-\phi_{x x}+\sin \phi=\lambda\left[\delta\left(x-x_{0}\right)-\delta\left(x+x_{0}\right)\right],
$$

as a not so realistic (though expected to exhibit the same features of more physical ones) example of application of their method, and, second, a change in the characteristic frequency of the system, which is put into mathematical form as

$$
\phi_{t u}-\phi_{x x}+[1+\varepsilon(x)] \sin \phi=0,
$$

with $\varepsilon(x)=\alpha \theta\left(x_{0}\right), \alpha \ll 1$. For the first case, they found that for $\lambda>0$ (respectively $\lambda<0$ ), solitons were decelerated (respectively acelerated) by computing the translation mode component of the first order correction to the soliton shape; after they pass over the impurity, they recover their original speed, and the only permanent change in them is a phase shift. On the other hand, the continuum modes give rise to a small distortion that is localized in the vicinity of the impurity, and, when the soliton is right over the impurity, to a slight modification of its shape. However, from the equations they found, the possibility that the soliton can be trapped by the impurity (when $\lambda>0$ ) arises. They showed that this indeed happens by a simple energy balance argument, in which the soliton is regarded as a classical particle; then, it must have enough kinetic energy to overcome the potential bar- 
rier of the impurity. By equating both energies the threshold velocity is found to be

$$
v_{\mathrm{thr}}^{2}=1-\left(1+\frac{1}{2}|\alpha| \tanh ^{-1} \sinh x_{0}\right)^{-2}
$$

$\alpha$ and $x_{0}$ being those defined above. The trapped soliton executes oscillatory motion, harmonic if its width is much less than the impurity extent, or anharmonic otherwise. More over, by further exploiting the Newtonian particle analogy, they were able to write down and solve the corresponding equations for the soliton center (this is not a proper collective coordinate treatment but a somewhat simpler way to proceed). Similar computations led Fogel et al. to conclude that, for the second perturbation (a generalization of this original problem has been recently considered by Fedyanin and Lisy, 1990), the soliton adjusts its shape and its speed to propagate in the new medium with larger characteristic frequency. Later, they checked all these perturbative results by means of numerical simulations (Currie et al., 1977), and found a good agreement between "theory" and "experiment" if the intensity of the perturbationwas not greater than 0.1 in dimensionless units. They also discovered that solitons were pinned in the absence of perturbation due to discreteness effects of the simulation: it can be shown by computing the energy with the expression (27) that the soliton must overcome a potential barrier to move from one lattice site to another adjacent one. This is not very important if the lattice spacing is much smaller than the soliton width, but if these lengths become comparable, then solitons are fastly pinned (with respect to this, it is very interesting to consult the papers by Bishop and Lewis, 1979, on pinning of narrow magnetic domain walls, or Peyrard and Kruskal, 1984, on simulations of sine-Gordon dynamics on highly discrete lattices; see also Willis et al., 1986, Stancioff et al., 1986, Boesch and Willis, 1989, and Boesch et al., 1989, for a collective coordinate study of this problem, recently extended by Braun and Kivshar, 1990, 1991). Having this in mind in order to perform simulations properly, they were able to show that the Newtonian particle analogy was a surprisingly good approach, that is nowadays widely applied as a simple way to estimate the perturbed dynamics of solitons.

Soon after those works, McLaughlin and Scott published their results on perturbative theory for the sine-Gordon system (McLaughlin and Scott, 1978). Their technique was the first one in using collective coordinates, as we have already mentioned, as well as in incorporating IST at a certain stage of the calculations. They studied a number of different perturbations of the system, each one of them chosen to represent certain physical effect on the system from the viewpoint of Josephson Junctions, which they describe in great detail. The general equation which they considered for the junction electrodynamics was 


$$
\phi_{t t}-\phi_{x x}+\sin \phi=-\alpha \phi_{t}+\beta \phi_{x x t}-\gamma-\sum_{i} \mu_{i} \delta\left(x-a_{i}\right) \sin \phi
$$

It is worth summarizing what all these terms represent in this context: $-\alpha \phi_{t}$ represents dissipation due to tunneling of normal electrons across the barrier between the two superconductors of the junction; $\beta \phi_{x x t}$ is another dissipativecontribution coming from electrons flowing parallel to the barrier; $\gamma$ is a distributed bias current providing energy input, and $\mu_{i} \delta\left(x-a_{i}\right)$ are local regions with high Josephson current, i.e., microshorts, thin spots, etc. (see Barone and Paternó, 1982 for a fully detailed description). This last term is a typically disorder one. Concerning it, McLaughlin and Scott wrote down the ordinary differential equations for the center and its speed when only a single impurity is present, and subsequently they integrated them numerically to get a picture of soliton evolution. Simultaneously, like Fogel et al., they benefited once more from the particle analogy to estimate the maximum bias current under which pinning can still occur at the microshorts, which happened to be $\gamma_{\text {thr }} \propto \alpha \sqrt{8 \mu+\mu^{2}}$. They compared it to experimental results (Scott et al., 1976) finding a very good agreement. They also computed the frequency of oscillations of pinned solitons around their pinning point, and show that these oscillations cannot couple to radiation emitted by the soliton, because the frequency of this last one is greater than unity and the oscillations occur with less frequency. It is worth remarking at this point that emission does take place due to the influence of nonlinear terms, leading to contributions from higher harmonics (Malomed, 1987a, 1987c; Kivshar and Malomed, 1988a).

The knowledge about point-like impurities in sine-Gordon systems remained essentially the same up to 1987 , with the exception of some isolated results, like those obtained by Paul $(1978,1979)$, who considered the problem of ferromagnetic domain wall pinning in easy plane ferromagnets with planar defects with similar techniques and calculations as those described above, and Malomed, (1985) who computed the energy spectrum and the total energy emitted by a kink weakly bound by a delta impurity. Next advances were done by Kivshar and Malomed (1988b) and Kivshar, Malomed and Nepomnyashchǐ (1988), who studied the opposite problem to that of McLaughlin and Scott, i.e., the presence of microresistors $(\mu<0)$ in the junction. The difference between both situations is that microshorts appear as repulsive potentials in the particle picture of soliton propagation while microresistors are attractive ones. In the mentioned papers, the threshold bias current for pinning by microresistors is computed and found to be much less than that of the microshorts, proportional to $\alpha^{3} \mu^{1 / 4}$. Besides, they took into account the dynamical effects of dissipation and wrote down an explicit expression (that turned out be very small) for the first order correction to the so far known results. In addition, they studied the general problem with dissipative inhomogeneities, given by an extra term $\delta \delta(x) \phi_{t}$ (a similar term but with $\phi_{x}$ was studied by Sakai et al., 1987, and Kosevich et al., 1987), and developed the 
corresponding perturbation theory to find the threshold for pinning under the combined influence of several of these perturbations. If $\varepsilon \gg \sqrt{|\varepsilon|} \gg \alpha$, the threshold bias force can be approximately written as $\gamma_{\text {thr }} \propto \alpha \varepsilon$. Kink scattering by a finite-size inhomogeneity was studied by Kivshar, Kosevich and Chubykalo (1988), who found that the main finite size effects were the increasing of the threshold bias under which solitons can be captured when the size $L$ of the inhomogeneity is increased, the possibility of capture of kinks by both repulsive and attractive inhomogeneities, and some hysteretical effects that could be checked experimentally. Finally, it is also interesting in this context the works by Braun and Kivshar $(1990,1991)$ who deal with the Frenkel-Kontorowa model (discrete sG) and discovered that there is some influence of discreteness in the collision of a kink with a impurity: for instance, the reflection of the kink is due to the potential barrier established by the discrete chain, taking place when the impurity mass is over some threshold proportional to the squared velocity and inversely proportional to the discreteness induced barrier; the kink diffusion is also varied by impurities and discreteness. They also show (Braun and Kivshar, 1991) that nonlinear impurity modes can be regarded as breathers trapped by the inhomogeneity.

To end these comments on point-like impurities, let us devote some words to the radiation problem. When the kink interacts with the impurity, it emits radiation in an amount that depends essentially on the amplitude of the perturbation and on the speed of the soliton. The first explicit formulas for radiation emission were obtained for Eilenberger (1977) for the case in which a simple term $\delta(x)$ is the perturbation. However, the most important part of this work has been done since 1985 and by means of the IST perturbation theory for solitons, which provide formulas to compute the energy density of the emitted radiation. Unfortunately, most of the time the integrals arising from that technique cannot be explicitly performed. Let us stress that even then they are by no means useless, because they can be numerically calculated for each specific choice of the parameters. What is always possible is to obtain asymptotic results for very small (but over the pinning threshold, of course) or very large speeds. Summarizing the obtained results, a general expression for the radiation emitted upon interaction with microshorts or microresistors was derived by Malomed (1988), who showed that for small velocities the total emitted energy (that propagates mainly backwards) was exponentially small, while for large ones it goes as $\sqrt{\left(1-v^{2}\right)}$. In a previous work (Malomed, 1985), he also derived the bias threshold for pinning due to the loss of energy through radiation, when pure dissipative terms are absent. For the general case (33), the computations can be found in Kivshar and Malomed (1988) and Kivshar, Malomed and Nepomnyashchin. The main outcome of those works is that the total emitted energy (which in this case goes both forward and backwards in a symmetric way) does not decrease with $v$ in the high speed regime, but instead grows as $\left(1-v^{2}\right)^{-1 / 2}$. This finding has to do with that by Kivshar, Kosevich and Chubykalo (1987b), 
where they used the fact that energy can only go to zero or infinity but not to a constant value in the high velocity limit, to prove that energy behaves as we have said because in the opposite case (radiation going to zero) there is an exact solution of the system, given by $\phi(x, t)=2 \pi \theta(x-t)$. The quoted papers provide enough detail on the computations and so we will not dwell on their description here. To finish, the review by Kivshar and Malomed (1989) mentions some other related computations about which we are not going to comment here; instead, we refer the reader to that paper.

\subsubsection{Lattices of impurities}

The current knowledge about more complicated problems, in which more than one impurity is involved, is less than that we have collected together in the preceding paragraphs for point-like impurities. Besides, it can be posed in several ways: think, e.g., of a lattice of point-like impurities (regular or random), or of a function of space or time (or both) modulating some terms in the equation. Let us first move to the kind of equation that McLaughlin and Scott (1978) proposed, Eq. (33), in which the whole term in the sum of delta functions is considered. This is relevant because the conclusions drawn about this model can be compared to experiments (Serpuchenko and Ustinov, 1987; Golubov et al., 1988). The question was first addressed, in the absence of any other perturbation, by Kivshar and Malomed (1985), who tried to compute the radiation emission of a kink propagating in a regular lattice of equal intensity impurities. Unfortunately, this problem is rather complicated and they were only able to obtain explicit expressions in some limit cases. In a first case, they studied the rarefied lattice, when it is possible to treat the interaction with each delta separately, and derived the radiated energy, most of which propagates forward; they also estimated the penetration length of slow kinks in such lattices. Secondly, they found that if the lattice is very dense, i.e., $a_{i}-a_{i-1}=a \ll 1$, the perturbation can be considered the same as that given by

$$
\phi_{t t}-\phi_{x x}+\sin \phi=\varepsilon \sin (\kappa x) \sin \phi,
$$

when $\kappa \gg 1$. Equation (34) has been studied by Mkrtchyan and Shmidt (1979), Malomed (1990a, including dissipation) and Malomed and Tribelsky (1990, they also addressed the same problem for trains of kinks, even densely packed arrays of them, including interference effects). If $\kappa \gg 1$ the emission of energy is exponentially small. The general result as obtained by Mkrtchyan and Shmidt (1979) is that radiation originates from the propagating kink when it evolves at a velocity over certain threshold related to the period of the modulation, $v_{\text {thr }}=\left(1+\kappa^{2}\right)^{-1 / 2}$.

Let us also mention some more papers dealing with the same topic, on whose description we are not going to enter, starting from two by Malomed (1987a, 
1987b), where several related perturbative terms are studied in great detail; there the interested reader can find a very complete account of all computations, which would be very difficult to summarize properly here. Malomed (1990b) computed the solitonic conductivity of these systems having in mind the presence of pinned solitons that have been previously captured at the inhomogeneities, and his computation was extended to more systems including continuous ones in Maiomed and Nepomnyashchii (1991). The problem of magnetic impurities has been considered in the paper by Ilyinikh and Shapiro (1990) by means of energetic analysis, and the bias current for depinning was obtained. Kivshar and Chubykalo (1991) have worked on the same question of magnetic inhomogeneitiesin Josephson junctions, finding a dependence of the current-voltage characteristicson the polarity of kinks. Bountis and Pnevmatikos (1990) and Bountis et al. (1990) applied the collective coordinates method to another problem, with only two impurities, and made a careful study of the resulting ordinary differential equations in terms of dynamical systems properties, describing the stability and instability of the pinning points. They report good agreement of their analysis compared with the simulations of the full partial differential equation. Similarly, a fair agreement has been also found recently between the theoretical predictions of IST perturbation theory by Kivshar, Kosevich and Chubykalo (1991) for the interference effects on the reflection coefficient of a soliton scattered by two point impurities, and the numerical simulations by Kivshar, Sánchez and Vázquez (1991). They show how the interference effects can give rise to oscillations in the dependence of the reflection coefficient on the distance between deltas for small speeds. These oscillations can be so large as to make the system practically transparent to the incident soliton for certain distances, and this does not happen for relativistic kinks.

We will finish these paragraphs devoted to lattice-like problems with some comments on what has become known as "supersolitons", though we are not mainly concerned in this review with multisoliton dynamics (in fact it is not proper multisoliton dynamics since one of the main assumptions needed for the computations is that there is a soliton chain which is rigid). The phenomenon has to do with the dynamics of groups of solitons in regular arrays of impurities, and we consider it because recently a large amount of work has been (and is still being) devoted to this problem. Moreover, the work has benefitted from the simultaneous information from theory, numerics and real experiments. A more or less thorough list of references on the subject would comprise Ustinov (1989), Oboznov and Ustinov (1989), Malomed (1990c), Malomed et al. (1990), Malomed and Ustinov (1990a, 1990b, 1990c) and Kivshar and Soboleva (1990a, 1990b). All these papers deal from one view point or another with the propagation of arrays of solitons in Josephson junctions with lattice impurities. Their main results are the following. In a first stage, as we already mentioned, the kink chain, whose expression is 


$$
\phi(x, t)=\pi-2 \operatorname{am}\left(\frac{x-\xi}{k}, k\right)
$$

where am is the Jacobi elliptic amplitude function, $0<k<1$ is its modulus (the usual kink is recovered when $k \rightarrow 1$; in the opposite limit one has a dense packing of solitons) and is an arbitrary constant, is assumed to be rigid. If so, and if the chain and the lattice have conmensurate periods (the lattice spacing is $a=p L / q$, $L$ being the period of the array of solitons), the minimum bias current that allows for the releasing of an initially pinned soliton chain can be computed by an energy balance, writing the corresponding Hamiltonians. In the limit $k \rightarrow 0$, and for arbitrary $p$ and $q$ the bias is $f_{\text {thr }} \propto q^{2} k^{2 q-3} / p$ (see Malomed and Ustinov 1990a, for instance). Later, Ustinov (1989) discovered numerically, Oboznov and Ustinov checked experimentally, and Malomed et al. (1990) showed analytically, that if the kink chain is allowed to rearrange itself instead of remaining totally rigid, a new phenomenon arises, which Ustinov called "supersoliton". They changed the constant $\xi$ (see above) into a function $\xi(x, t)$ and using again the Hamiltonian of the sG system they found an equation for this phase $\xi(x, t)$, which on its own supports an exact kink-like solution (though it is not integrable). This kink-like solution is straightforwardly interpreted as a "hole" in the soliton chain (or as an extra kink in the chain if its polarity is the opposite); in connection with this remark, this interpretation has to do with the similarity of the array of solitons in a periodic lattice of impurities with the Frenkel-Kontorowa (Frenkel and Kontorowa, 1939) model of elastically interacting particles on a periodic substrate potential. Supersolitons would then be something like the solitons of the Frenkel-Kontorowa model, that arise as local variations of the particle density when the period of the chain of particles differs slightly from that of the underlying potential; these solitons are the dislocations the Frenkel-Kontorowa model intends to present, and, as we have said, would be more or less equivalent (Ustinov, 1989) to supersolitons, the fluxons in the array being kind of particles in a elastic chain. Finally, they simulated numerically the partial differential equation and also carried out experiments in specially designed Josephson junctions, that were compared to the intensity-voltage curves they computed from their previous results. The agreement between the three approaches was found to be excellent. The last results that should be included are the recent ones by Kivshar and Soboleva (1990a, 1990b) on the existence of envelope supersolitons, aside from the kink ones we have been talking about, in layered Josephson structures, which involve a system of coupled sG equations.

\subsubsection{Fluctuations and continuously random impurities}

In the previous paragraphs we have mentioned some perturbations introduced as a continuous modulation of the parameters of sine-Gordon systems, given by trigonometric functions. We now aim to summarize what has been done 
concerning the problem of continuous, but random, impurities, which is rather different from the ones we have described up to now. The difference between this sort of perturbation and lattices of point-like impurities is rather obvious: unless the lattice is so dense as to allow its substitution by a function, the properties that one can expect for the system under these deviations from order should be quite different, because if affected by a lattice, only a finite number of space points are influenced instead of an infinite number (the whole line, or an interval). It will show also features not similar to those of functions, because we have now to deal with stochastic processes and, then, sometimes we will have to average on their realizations, sometimes we will be interested in one realization, but in any case the description become probabilistic.

The work on stochastic perturbations (see Bass et al., 1988, or Rodriguez-Plaza and Vázquez, 1988, for previous topical reviews) begun in the decade of the 80's. More results have been obtained for temporal stochastic perturbations, because the mathematical problems that one has to get rid of when studying spatial ones are really hard. All the same, to our knowledge, the first such study is due to Mineev, Feigelman and Shmidt (1981), who among the family of problems

$$
\phi_{t t}-\phi_{x x}+\sin \phi=\varepsilon f(x, t) R[\phi],
$$

considered the one with $f(x, t)=f(x), f$ being a gaussian white noise with correlation $\left\langle f(x) f\left(x^{\prime}\right)\right\rangle=\delta\left(x-x^{\prime}\right)$, and $R[\phi]=\sin \phi$. Using the mode decomposition, they proved a formula for the spectral density of the emitted radiation, and show that the total energy, which can be approximately computed in the limits of small and high speeds, has a maximum at a certain intermediate value of the kink velocity; besides, they describe characteristic hysteretic effects due to the so induced dissipation in the characteristic curves of the Junction. Later, Bass and Sinitsyn (1982) studied the problem starting from a three-dimensional view point, and particularizing their results for several limiting cases, concerning the longitudinal and transverse directions, but for general $f$. For $1 \mathrm{D}$ longitudinal small scale inhomogeneities, they found that the first correction was localized around the center of the kink and that it was proportional to the cube of its velocity, (to its square for large scale fluctuating inhomogeneities).

In 1985, the IST perturbation theory entered the field of stochastic perturbations (stochastic perturbation theory) through the papers by Bass et al. (1984, 1985, 1986) and Kivshar et al. (1986; this paper has some parts devoted to the Landau-Lifshitz equation, 1987). They wrote down the adiabatic equations (i.e., the equations for the collective coordinates: the kink center and its velocity) and subsequently they found a Fokker-Planck-like equation for the corresponding probability density, for a noisy term with correlations $\left\langle f(x, t) f\left(x^{\prime} t^{\prime}\right)\right\rangle=$ $B\left(x-x^{\prime}\right) \delta\left(t-t^{\prime}\right)$. A parenthetical remark is that if the disorder is purely spatial, it is not at all possible to write a Fokker-Planck equation. However, some progress 
can be made (Gredeskul et al., 1991) in this case by means of studying the behavior of the temporal intervals between randomly located impurities instead of the spatial variable directly. Coming back to the Fokker-Planck equation, it can be solved in the WKB approximation and then the average values can be computed. They studied an external force, i.e., $R[\phi] \equiv 1$ in Eq. (36) (Bass et al., 1985) and $R[\phi] \equiv \sin (\phi), \sin (\phi / 2)$ (Kivshar et al., 1986, 1987) and presented some calculations for the limit of small and large correlations lengths of the spatial part of the noise. For the former, the kink is slowed down in mean value or it undergoes an accelerated oscillatory motion if it was initially at rest. The same happens in the large correlation limit but the dispersion of the center position grows linearly instead of cubically as in the previous case. The radiation emission was computed in Kivshar et al. (1986, 1987). Finally, in Pascual et al. (1989), the problem of spatial white noise is addresed from the numerical point of view (see also Sánchez and Vázquez, 1989, for a study of the stationary stochastic configurations of such a system) as well as from the analytical one, by studying the adiabatic equations for short times. They discovered by both means that the response of a kink at rest to the switching on of the noise was asymmetric, the kink moving to the positive part of the $x$ axis.

We end the sine-Gordon part of the survey with some results concerning fluctuations, i.e., perturbations for which the stochastic term is not depending on the space (and thus constituting a type of dynamical disorder). These kind of equations are more amenable to analytical approaches (for instance, it is rather easy to derive collective coordinates equations, that now are Langevin equations, through the conserved quantities techniques) and then they are suitable to check numerical simulations in the weak noise regime, to ensure the absence of numerical, non-physical chaos. Pure additive noise was considered in the work by Salerno et al., (1984) playing the role of a coupling to a heat reservoir. Kinks and phonons were shown to be thermalized, the first having $k_{\mathrm{B}} T / 2$ average energy per mode, and the second $k_{\mathrm{B}} T$; the diffusion constant of brownian motion of kinks was also computed and proved to be the same as that of a point-like particle. Later, Marchesoni and Vázquez (1985) numerically observed the evolution from an initial kink to kink-antikink pairs due to strong additive or multiplicative noise, with no blow ups. A more detailed study can be found in Pascual and Vázquez (1985), who were interested in the collective coordinates evolution of the kink, which they calculate analytically and numerically for additive or linearly multiplicative (i.e., $R[\phi]=\phi$ ) finding a good agreement between the outcomes of both procedures. This allowed them to separate weak and strong noise by a definite threshold checking whether the perturbative predictions were verified or not. Their results were fully confirmed by simulations with a much larger number of realizations (Biller and Petruccione, 1990; Petruccione and Biller, 1990). On the other hand, in the paper by Kaup and Osman (1986) a singular perturbative expansion was developed, as we mentioned in the corresponding section. They used it to make a thorough study of the thermal sine-Gordon problem, computing 
the same quantities that had been already obtained as well as some other ones, verifying so their method, and proving that the limit of large damping for sine-Gordon systems is not the so called overdamped sine-Gordon system of Kaup (1983). Bass et al. (1986) use similar techniques as above to work with gaussian white noise or Poisson noises, obtaining general results, like that for odd $R(\phi)$ the shape and velocity of the kink are not altered in the average, while they do are modified for even $R(\phi)$. Poisson noise was seen to accelerate or decelerate solitons depending on their sign for odd perturbations, and a general expression was obtained for even ones. The problem of kink diffusion has been recently addressed by Krug and Spohn (1989), who find anomalous exponents for driven and damped sine-Gordon systems, given by dispersion growing as $t^{2 / 3}$; Willis (1989) came back to it and compare his analytical results to some experiments, and Marchesoni and Willis (1990) described two different regimes, anomalous and viscous, depending on the possibility of neglecting interaction between solitons and phonons or not, that is related to the temperature of the system. To end with, Ivanov and Kolezhuk (1990; see also references therein) have made a thorough work on this question for several NKG models and again reported these two kinds of diffusive processes, describing them in full detail.

\section{2. $\phi^{4}$ model}

\subsubsection{Generalities}

The amount of research that has been done up to date in the $\phi^{4}$ equation and perturbed versions of it is actually much less than that concerned with sG problems. Probably two of the main reasons for this are the non-integrability of the $\phi^{4}$ model and the smaller range of applications it has. However, it must not be concluded that the model is not important, because it has served to develop special techniques not based on IST as well as to provide a lot of knowledge on numerical simulations of evolution equations.

The $\phi^{4}$ equation is obtained from the Lagrangian (24) putting $V(\phi)=$ $\left(1-\phi^{2}\right)^{2} / 4$, or, in its discrete version, from the Hamiltonian (27) with the same substitution. The subtleties involved in this change is that the sG potential has an infinitely degenerate minimum, located at $\phi=2 k \pi, k$ any integer, and the $\phi^{4}$ potential is a double well one with a doubly degenerate minimum at $\phi= \pm 1$. This breaks, of course, the possibility of having multisoliton solutions, that arise in $\mathrm{sG}$ systems from the connection between any of the different values of the minima; in the $\phi^{4}$ model only the two existing minima can be connected, and the most complicated outcome of that is given by a sequence kink antikink alternatively. However, it must be also said that this is not the root reason for non-integrability. Think for instance of the DsG model, Eq. (4), which has an infinitely degenerate ground state but has no $N$ soliton solutions.

With regard to $\phi^{4}$ model properties, energy, momentum and topological charge are conserved in the $\phi^{4}$ model, but as far as we know there is no other conserved 
magnitude. Soliton-like solutions (soliton is used as a loose terminology) are given by

$$
\phi(x, t)=\tanh \left[\frac{\gamma}{\sqrt{2}}\left(x-v t-x_{0}\right)\right]
$$

and there are no stable breathers as Segur and Kruskal showed (1987; see in addition Boyd, 1990 for a current report on this problem), though small amplitude, weakly unstable nonlinear wave packets can be approximated by NLS envelope solitons (Newell, 1985, Remoissenet 1986). In fact, most of the knowledge on the unperturbed $\phi^{4}$ model comes from numerical simulations, which have been performed by a large number of researchers (see Schneider and Stoll, 1978, and references therein; Ablowitz et al., 1979; Campbell et al., 1983; Combs and Yip, 1983, 1984; Kunz and Combs, 1985; Campbell and Peyrard, 1986; Campbell et al., 1991) and a very interesting phenomenology has been reported. The most curious phenomenon is that when a kink collide with an antikink (obviously kink-kink collisions are forbidden if both are of the same polarity) they annihilate each other if the collision takes place under a certain velocity, or rebound and separate if it happens over that velocity; however, there are some windows in the annihilation range of velocities in which the separation between kinks follows a series of integer number of bounces (Campbell et al., 1983, Campbell et al., 1991), due to certain resonance phenomena. This resonance is due to a change in the spectrum of linear excitations with respect to the one we have already described for the sG system: there is one extra discrete eigenvalue, which corresponds to the so-called internal mode. This internal mode (that has been related to the roots of non integrability, see Bogdan et al., 1990) is always localized in the vicinity of the soliton, is related to shape distortions, and plays a fundamental role in any interaction of solitons with each other, with radiation or with impurities (see Campbell et al., 1983, for a thorough account on this mode).

Let us conclude these general remarks by mentioning applications related to the model. It was born as a prototype of scalar field theory (Dashen et al., 1974; see also Moshir, 1981). When it was proposed in condensed matter for the first time, it was intended to describe structural phase transitions (Aubry, 1975, 1976; Krumhansl and Schrieffer, 1975), and dynamics of domain walls in ferroelectric and ferromagnetic materials (Bishop et al., 1977; Collins et al., 1979; Currie et al., 1979; Imada, 1981, these last two works contain comparisons with real systems). Later it was used in connection with polymer physics, describing mismatches in the carbon bonds (Rice, 1979; Rice and Timonen, 1979; Mele and Rice, 1980; Jackiw and Schrieffer, 1981) very accurately (Guinea, 1984, made a more precise calculation from the quantum viewpoint and found that the results were very similar). Finally, it was proposed as a model for hydrogen bonded chains (Gordon, 1987, 1989, this last paper being another interesting example of 
application to a real physical system, namely ice; Pnevmatikos, 1987), but it was found to be not very accurate; nevertheless, current models usually benefit in one form or another from the knowledge of the model.

\subsubsection{Perturbed $\phi^{4}$ models}

Of course, the work on the subject of perturbations of $\phi^{4}$ models has been faced from a completely different viewpoint than that on the $s G$ systems, because many of the problems that can be directly solved or at least accounted for to some extent in the latter are very difficult questions in the former, because of its non-integrability. So, the first papers on this topic deal with the problem of nonlinear interactions between kinks and phonons. These are the articles by Wada and Schrieffer (1979) and Theodorakopoulos (1979); in them, it is shown that phonon-kink interaction yields an induced phase shift in the kink, computing direct perturbative expansions. They were also able to compute the diffusion constant (although following different approaches) due to the collision of the kink with the thermal phonons that are always present in lattices at a finite temperature. Next efforts in this line were done in two works by Collins et al. (1979) and Currie et al. (1979). They considered the general equation

$$
\phi_{t t}-\phi_{x x}-\phi+\phi^{3}=-\alpha \phi_{t}+F(x, t)+f(x, t),
$$

where as usual the term in the first derivative represents dissipation, the term $F$ is an external force and $f$ is a random process, that subsequently they fix to be delta correlated both in time and in space. They proved kinks to be stable and then computed once more their diffusion constant, the limit velocity achieved by them and the structure factor of the system, which they compared very successfully with experiments in uniaxial displacive ferroelectrics. Recently, a new application of Eq. (38) was considered by Ji-Zhong Xu (1990), computing the same quantities for hydrogen-bonded chains. Further progress was made in the works by Bass et al. $(1984,1986)$ which we already mentioned in the sineGordon section. They extended their computations for both models treating the general NKG equation. The results they obtained are essentially the same as in sG. A more general $\phi^{4}$ model was studied by Pnevmatikos et al. (1986): in their research they considered the possibility that the anharmonicity were also quadratic, and included forces and dissipation, considering even envelope wave packets dynamics, mainly through simulation.

We will close the section with a summary of the most recent results. Beloshapkin et al. (1989) performed numerical simulations on the $\phi^{4}$ model searching for the existence of regular configurations of solitons, but found that these structures were unstable under fluctuations in a fashion opposite to that of the Frenkel-Kontorowa (sG) model. This happens via the fluctuations on the depth of the potential wells which arises due to temperature effects, and so the threshold for the mutual kink-antikink anihilation is lowered. Fraggis and 
Pnevmatikos (1989) and Fraggis et al. (1989) studied the consequences of the presence of an impurity in the discrete $\phi^{4}$ model, the impurity being characterized for a different mass. They made an analytical study, computing the vibration mode corresponding to the impurity, which is localized in a certain length which they also obtain. Subsequently, by means of numerical simulation, they consider the excitation of such mode by a kink propagating in the chain. Only the amplitude of the impurity vibration was found to depend on the soliton energy. They also showed that kinks are reflected (respectively transmitted) if the impurity mass is larger (respectively smaller) than some critical value. Bogdan and Kosevich (1990) concerned themselves with the study of localized modes in a number of models, including the $\phi^{4}$ one too. They compute analytically and numerically the spectrum of these excitations and show the applicability of the lattice defect theory to these computations. They account for the induced switching on of the localized vibrations by external forces. Lastly, Kivshar, Zhang Fei and Vázquez (1991) have discovered that the picture of kink-impurity scattering is more complicated than that of the works by Fraggis and Pnevmatikos (1989) and Fraggis et al. because of the presence of resonant windows in a similar fashion than in kink-antikink collisions.

The last paragraph in this section is devoted to a series of works (RodríguezPlaza and Vázquez, 1990; Sánchez and Vázquez, 1991; Konotop et al., 1991; Sánchez et al., 1991), in which a thorough study of the influence of random fluctuations on the $\phi^{4}$ model was carried out. Rodríguez-Plaza and Vázquez (1990) treated the cases of additive and linearly multiplicative white noises, writing the adiabatic equations for the collective coordinates and the associated Fokker-Planck equation. From this equation they obtained the equations for the mean values of the coordinates (the same as obtained by Bass et al., 1986 with a different procedure), which they found to be in good agreement with numerics, and computed exactly the probability density in the additive case. Additive noise produced a displacement of the energy center and an acceleration of the kink independently of the initial conditions, in contrast with multiplicative noise that depends on them. Later, Sánchez and Vázquez (1991) studied the nonlinear multiplicative case (which represents the wells depth fluctuation of Beloshapkin et al.) numerically as well as in the framework of collective coordinates. They found the regimes of weak and strong noises, and show that the energy of the system grows exponentially with an exponent that depends almost linearly on the noise strength. In addition, they reported anomalous diffusion of kink center under strong noise. Radiation was found to appear with a dependence on the kink velocity similar to that of the $\mathrm{sG}$ system. The numerical work was generalized in Sánchez et al. (1991) where they also show in great detail how the numerical procedure is as accurate as to reproduce fairly predictions for energy growing by Parrondo et al. (1990). They included dissipation effects, which were mainly a raising of the weak noise threshold, and boundaries between perturbed and unperturbed noises, which were able to get kinks pinned very fast. Finally, 
Konotop et al. (1991) developed a secular perturbation theory for this problem using the collective coordinates as counterterms, and explain the numerical results and the appearance of radiation with quite good agreement.

\section{Nonlinear Schrödinger Equation}

\subsection{Generalities}

The nonlinear Schrödinger equation, Eq. (5), has been given that name in view of the fact that it shows the same structure of a Schrodinger equation with a potential that depends on the amplitude of the probability density. However, it has neither a special relationship with the Schródinger equation, nor any quantum meaning, other than the name. It does play a significant role in many physical situations; to be specific, in dispersive systems where no dissipation occurs, when one is aimed to study small amplitude waves or envelope solitons. It derives from the Lagrangian

$$
\mathscr{L}=\int_{-\infty}^{\infty} d x\left[\frac{i}{2}\left(\phi \phi_{t}^{*}-\phi^{*} \phi_{t}\right)+\left|\phi_{x}\right|^{2}-|\phi|^{4}\right],
$$

and its most important solution is the two-parametric, envelope soliton (15) we wrote down in the Introduction. Notice that Eq. (5) also allows for the propagation of plane waves. These planes waves have been show to be unstable (unlike those of the NKG equation, that also exist but are stable) due to the effects of sidebands of their frequency; this is the so-called Benjamin-Feir instability (Benjamin and Feir, 1967; Benjamin, 1967). As a consequence, they decay into localized objects, like nonlinear wave packets or solitons. So, solitons will have the protagonist part in the dynamics of these systems.

The NLS equation is integrable, as shown by Zakharov and Shabat (1971); their work was indeed very important because the Lax pair they proposed to solve the NLS equation via IST was later generalized to include as particular cases the KdV, $\mathrm{mKdV}$ and $\mathrm{sG}$ equations (Ablowitz et al., 1973, 1974; see also Dodd et al., 1982). It is worth mentioning at this point that Balakrishnan (1985) has extended this formalism (usually referred to as AKNS-ZS, after the initials of their discoverers) to a particular perturbed NLS equation derived from a classical inhomogeneous Heisenberg chain in the continuum limit, this being a case of solvable disordered systems very different from those we considered in the second section, because it is fully meaningful. Due to its integrability, the unperturbed NLS equation (5) has an infinite number of conserved quantities; the most physically relevant are the energy, the momentum and the charge (also called "number of quasiparticles") that we mentioned when dealing with numerical techniques. Concerning its connection with physical applications, the NLS equation can be deduced from a large bunch of physical problems in different ways (see, e.g, Tappert and Varma, 1970; Dodd et al., 1982; Remoissenet, 1986; Spatschek, 
1987; Remoissenet, 1989). It is easy to suppose from these comments that the NLS equation has a very broad range of applications. It has achieved an enormous success in two specific contexts: plasma physics, to be precise propagation of Langmuir waves (Ichikawa et al., 1972; Shimizu and Ichikawa, 1972; Fried and Ichikawa, 1973; see Spatschek, 1987 for a review), and nonlinear optics, describing self-modulation and self-focusing of light in a Kerr type nonlinear medium (Chiao et al., 1964; Kelley, 1965), specially in optical fibers (in this application the role of $x$ and $t$ variables is interchanged, see Hasegawa and Tappert, 1973a, 1973b; Mollenauer and Stolen, 1984; Haus and Islam, 1985; see also the book by Hasegawa, 1988). Aside from these subjects, the NLS equation arises in Ginzburg-Landau theory of superconductivity (de Gennes, 1966), nonlinear surface waves in dielectrics and semiconductors (Gorentsveig et al., 1990; Kivshar, 1991a), Davydov solitons (Davydov, 1985), flexural modes of thin shells (Wu et al., 1987), surface waves in liquids (Denardo et al., 1990) and in discrete systems like atomic lattices (Tsurui, 1977), magnetic chains (Lakshmanan, 1977; Corones, 1977), electrical networks (Muroya et al., 1982), or two component lattices (Yajima and Satsuma, 1979, Pnevmatikos et al., 1986).

\subsection{Background: perturbations of linear Schrödinger problems}

Although, as we have stated above, the nonlinear Schrödinger equation has nothing to do with quantum problems described by linear Schrödinger operators, the study of disorder in both kind of systems is indeed deeply connected. This is the reason why we think that we should briefly collect some results on the linear problem before entering nonlinear ones. The linear Schrödinger equation has been dealt with extensively in connection with electronic transport properties in Condensed Matter Physics (see, for instance, the books by Ziman, 1979, or Economou, 1983, or the reviews by Thouless, 1974, or Lee and Ramakrishnan, 1985). The most important notion in order to understand the properties of electrons in disordered systems is that of localization (Anderson, 1958). In his celebrated work, Anderson showed that the electronic states at the center of the band must be localized (i.e., their probability density must decay exponentially with the distance to some point, behaving like $\exp (-x / L))$ if the energy of the individual atomic states varies at random over a range somewhat greater than the width of the band produced by overlap between adjacent atomic orbitals. In other words: if the disorder is strong enough, electrons are unable to propagate and remain localized in a region of certain radius or localization length (the exponent $L$ ). This result becomes stronger when restricted to one spatial dimension: all the normal modes and eigenfunctions of a disordered linear chain are localized (Mott and Twose, 1961; Makinson and Roberts, 1962), a property that can be proved to hold for all types of excitations in all the standard models of one-dimensional disorder (Matsuda and Ishii, 1970). In the last years, the research on this problem, that, mathematically speaking, is related to the nature of the spectrum 
of self-adjoint random operators, has benefited greatly from intensive interaction between stochastic analysis and quantum theory. So, a number of results have been obtained concerning energy values accesible to the particle, properties of stationary states, time evolution and transport properties of the system. Very recent and comprehensive surveys can be found in Kunz (1980), Kirsch (1985), Sinai (1987), Lifshitz et al. (1988) and Ping (1990). Some work has also been devoted to time dependent problems; thus, Ovchinnikov et al. (1975) and Madhukar et al. (1977) addresed the Schrödinger equation with random Gaussian potentials, delta-correlated in time. Other results, for more general Markovian processes, have been obtained by Pillet $(1985,1986)$. This kind of problems appear in Condensed Matter Physics when one tries to account for fluctuations in disordered media (Paquet and Leroux-Hougon, 1984), and the main questions that are posed in these situations are the stability and the energy growth in the system, as well as the existence of bound states. Nevertheless, in spite of all this work on the Schrödinger problem, the question of localization of classical waves (we mean, do classical waves propagate through unchanged, or are attenuated by disorder? If they are attenuated, what is their localization length?) have been the subject of the interest of physicists only recently. There are some classical works (Chernov, 1960; Barabanenkov et al., 1970) but the growing of research in the field has taken place in the last seven years (see, e.g., Lifshitz et al., 1988, or Economou, 1990, and references therein; see also the thorough topical review by Freylikher and Gredeskul, 1991). This is even more surprising when one realizes the number of practical applications of this problem in fields like geophysics, communications, optics, etc. The current situation is more so concerning nonlinear problems, and in fact we must say that this field is still starting up and much more questions have been posed than those that have been solved. In the next section we will try to summarize from this viewpoint of localization theory what have been the main achievements concerning nonlinear systems.

\subsection{Perturbed nonlinear Schrödinger equations}

The work on nonlinear Schrodinger systems from the viewpoint of localization theory is mainly concerned with the influence of nonlinearity in the linear systems features, i.e., with determining whether nonlinearity changes or not the localization property. The first research in this direction was carried out by Devillard and Souillard (1986), who considered the problem of a nonlinear disordered segment between linear free ends and study its stationary solutions of the form $\exp (i w t) f(x)$. The choice of such so called semilinear model allows them to start from linear waves at the ends and study the transmission changes due to the central nonlinear part, in which they placed a potential consisting of piecewise linear potential steps of random length and height. They addresed the problem in a rather mathematical framework, and by that means they were able 
to prove several interesting results; furthermore, they also performed numerical simulations on the model. From their work, it can be concluded that the problem is different depending on the way the boundary conditions are chosen. This means that the problems for the transmission coefficient for a certain input power (fixed input problem) and the transmission coefficient that provides a certain output power (fixed output problem) are not the same: in the former, transmittivity decays with the length $L$ of the nonlinear slab as an exponential law, while, in the latter, transmittivity decays as a power law, that they numerically found to be simply $L^{-1}$. Later, Doucot and Rammal considered the same problem in a letter (1987a) and two vast papers (1987b; the first one is devoted only to the linear problem but using the same techniques) with a gaussian white noise potential, constant in time. They developed an embedding approach to deal with the problem and showed that in their situation, the transmittivity of linear waves decayed as $L^{-\alpha}, 1<\alpha<2$ in the fixed output problem. They also proved that multistability happened when the conditions were that of the fixed input problem; this is to say, several possible outputs can be originated by the same input. In connection with this stability problem, it is worth mentioning also the work by Knapp et al. (1989), where they investigate the semilinear equation with a bounded, stationary, zero-mean random potential from a more optical viewpoint. They carried out an exhaustive program of numerical simulations and described the different behaviors of the transmission coefficient as a function of the problem parameters. So, with respect to this semilinear approach, the panorama is rather confusing and most of the questions remain open, as it happens even in the linear case (see, for instance, Knapp et al., 1989, or Economou, 1990).

Let us now move to the problem of transmission of solitons, which was first considered by Qiming et al. (1988b) as an extension of previous related work (Qiming et al., 1988a) on anharmonic lattices. They study by numerical simulation the usual harmonic lattice including cubic and quartic anharmonicities, which has small amplitude excitations that are quite accurately described by the NLS equation, and also allows for propagation of kinks. Inside the chain a segment is disordered isotopically, its atoms having a mass value with probability $p$ and a different one with probability $1-p$. The transmission coefficient for such a system is defined as the ratio of the total energy contained in the part of the chain beyond the disordered slab to the total energy on the system. They found that this coefficient is decreasing as $L^{-1 / 2}$ (short cubically anharmonic systems yielded $L^{-3 / 2}$, but if they were long enough the other dependence was recovered). They compared their simulations with two limiting cases: in the independent scattering limit, in which the concentration of impurities is low (hence the mean distance between them is larger than the soliton size and one is allowed to neglect interference effects), they were able to reproduce analytically the numerical results; on the other hand, in the linear approximation, which holds for very long systems, they computed the transmission coefficient for linear wave packets and 
again found the same dependence. So, they were forced to conclude that the scattering behavior of linear wave packets and solitons is approximately the same. Further related numerical simulations were provided by Caputo et al. (1989) on the Ablowitz-Ladik discretization of the NLS system. They started from linear waves that were launched against a randomly disorderd slab and discovered that they were much more quickly instabilized than in homogeneous systems, evolving into a bunch of solitons that considerably improved the transmission properties of the systems. Similar results concerning acceleration of instabilization processes have been found by Peyrard and Bishop (1990) and Kivshar (1991). Up to our knowledge, the last result on this complex problem of soliton localization is that of Kivshar, Gredeskul, Sánchez and Vázquez (1990, 1991; this last paper considers also the problem for linear wave packets, that are shown to decay with a power law). They worked on the NLS equation perturbed by an array of point-like impurities. In their calculations they started from the previous expressions obtained by Kivshar et al. (1987a) for the transmission coefficient of a single impurity by means of IST perturbation theory for solitons (static solutions for such an equation have been recently obtained by Pushkarov and Atanasov, 1990). They also considered in that work the interference effects arising in scattering from two impurities, similar to those described for sine-Gordon systems above (Kivshar et al., 1991), and included some computations on lattices of impurities. With this background, and within the same independent scattering approach of Qiming et al. (1988), Kivshar et al. (1990, 1991) obtained the integro-differential equations governing the energy and the number of quasiparticles in the soliton as functions of the distance traveled in the disordered lattice. They found that some threshold value for the nonlinearity exists, such that "more nonlinear" solitons were able to rearrange themselves and to propagate undistortedly in the system. This is in agreement with the outcome of the recent numerical simulations of Bourbonnais and Maynard (1990), who showed that energy diffusion in one-and two-dimensional lattices takes place beyond the localization length for enough strong anharmonicity, but it does not depend on the amount of disorder. To end this section, we will mention some problems which arise more specifically in nonlinear optics. This research area is growing quite rapidly nowadays due to the richness of observed phenomena, the possibility to check computations experimentally (in this sense, optical fibers play the same role in NLS theory than Josephson junctions in sG theory), and the importance of technological applications. Solitons in nonlinear single mode optical fibers were observed for the first time by Mollenauer et al. (1980; see also Mitschke and Mollenauer, 1987, and Kodama and Nozaki, 1987); recently, they have been transmitted through $6000 \mathrm{~km}$ of fiber (Mollenauer and Smith, 1988), a fact of enormous consequences in the world of communication. However, these solitons exist only if the coefficients of $\phi_{x x}$ (remember that now $x$ plays the role of time), i.e., the group-velocity dispersion, and the coefficient of the nonlinear term, that arise from the Kerr effect, have the same sign. When the signs are 
opposite, the equation still remains integrable, and has another type of solitons called "dark solitons" (on the contrary, usual ones are called "bright") given by

$$
\phi(x, t)=\phi_{0} \frac{[(\lambda+i v)+\exp (X)] \exp \left(2 i \phi_{0} t\right)}{1+\exp (X)}
$$

with $X \equiv 2 v \phi_{0}\left(t+2 \lambda \phi_{0}^{2} x\right)$, and $v \equiv\left(1-\lambda^{2}\right)^{1 / 2} ;-2 \lambda$ is the soliton velocity and $v$ is its only governing parameter, that characterizes the soliton intensity. These solitons, that are localized nonlinear excitations of the form of rapid dips in the intensity of a carrier wave background, have been recently found experimentally (Krökel et al., 1988, Weiner et al., 1988), fifteen years after their theoretical prediction (Hasegawa and Tappert, 1973b; Zakharov and Shabat, 1973). The study of these excitations in disordered systems is being started now, following similar studies for bright solitons. In them, random initial conditions are given for the NLS problem, that can simulate the fluctuations induced by the medium and have been studied by a number of researchers (see, e.g, Vysloukh et al., 1987; Konotop, 1990; Gredeskul et al., 1990; Konotop and Vekslerchik, 1991, and references therein). These last two papers (Gredeskul et al., 1990; Konotop and Vekslerchik, 1991) deal with the same kind of studies for dark solitons, discussing with the help of IST perturbation theory the dynamics of solitons evolving from initial conditions as well as the possibility of generation of new ones.

\section{Closing Remarks}

We hope that along the paper we have given the reader an overall panorama of the systems comprised by the study of Nonlinear Wave Propagation in Random Media, as well as the techniques that are most often used as a basis for work on such systems. We also hope that, by means of the examples related to NKG and NLS problems, he will have offered a flavour of the things that are amenable to analytical or numerical calculations, and not only that, but also the kind of quantities that are as interesting as to make useful any controled approximate prediction one can do about them. If so, we would have succeeded in providing the non-expert with the fundamental ideas required to start the study in depth of this fascinating subject.

Were we requested to select among the ones we have reviewed a few topics to stress as the most important, we would pick two of them. The first is the synergetic approach to the study of nonlinear wave dynamics, combining all viewpoints: experimental, theoretical and numerical. We have already said this several times. Now, at the end of the paper, we would really like to have brought forward enough evidence that this is so. Investigation is more often than not frustrated and frustrating if one of the three aspects is forgotten. Thus, it can happen that the theoretician does not even know what he must compute, because he does not have any idea of the kind of phenomena a new perturbation can 
originate; the computational physicist can be making enormous amounts of simulations (and spending enormous amounts of money, too!) without making any interesting contribution to the understanding of nonlinear physics, because he is not computing crucial properties of his system; a similar situation can occur to experimentalists. Besides, both theoreticians and computational physicist must pay a lot of attention to experiments, to study equations and problems as close as possible to reality.

The other main conclusion we want to offer the reader is the extremely rich world of phenomena that arises from the study of nonlinear waves. The behavior of solitons in each system depends essentially on the type of excitation it is (see related comments by Kivshar, 1991b), and not so much on the exact system we are considering. For instance, small amplitude wave packets propagating in NKG models are much more similar to NLS solitons than to NKG ones, because both are envelope solitons. We can classify solitons by considering their robustness. The weakest class is that of dynamical solitons, that actually look like linear wave packets in linear systems; for instance, when propagating in a disordered model, both excitations exhibit power law dependence of their transmission coefficients on the traveled distance. Next, we would have to place envelope solitons. They are able to propagate even in the presence of disorder, at least if they are nonlinear enough. However, they suffer an initial distortion, and, even more, when the system size increases very much, they finally become, for practical purposes, linear wave packets, and therefore they get localized. At last, topological solitons prove themselves to be the most robust. In many situations the effect of perturbations on them can be accounted for by a simple collective coordinate treatment, which amounts to consider them as particles. This is related to the strength of their structure, at the root of which is their topological nature, protecting themselves from annihilation even when they are pinned by disorder.

Indeed, Nonlinear Wave Propagation in Random Media is a very exciting field, and it is not difficult to imagine that the future will be even brighter than now. There are lots of challenging problems waiting to be solved; some of them have not been faced yet. Think, for instance, of the recently open areas to which we devoted the end of NKG and NLS sections: supersolitons in Josephson junctions, dark solitons in fiber optics. The necessity for technological progress will require a lot of effort on these and similar topics. But they are only the newest discoveries in the field, and there are so many other problems, like the clarification of the question concerning the transmission coefficient in inhomogeneous NLS systems, both for linear wave packets and solitons, the interference effects from more than two impurities and for continuous impurities, interaction between nonlinear excitations and impurity modes, nonlinear equations under spatial random perturbations, general (colored) stochastic perturbations both in time and space... As we stated above, the trend should always be to approach as much as possible the conditions of real physical systems. 
Another reason for the optimistic point of view we are supporting here is that the number of applications of nonlinear physics is growing every day. Every day, pure (non-numerical) experiments are designed, and the experimental contribution to nonlinear knowledge is getting a place on his own. We are at the beginning of an era of nonlinear biophysics and biochemistry: a rapidly increasing group of researchers is changing their main interests to topics like excitations in and denaturation of DNA and other nucleic acids. Also polymer physics is becoming more and more influenced from nonlinear perspectives. The necessary inclusion of quantum effects when dealing with solitons at microscopic level is being currently addressed. On the other hand, as we announced in the introduction, nonlinearity is about to enter the up-to-date linear field of fractals. And, it goes without saying, nonlinear physics in more than one dimension is starting up too. Solitons, nonlinear physics, are getting a fundamental tool, at the very root of cross disciplinary physics. It is this open-minded, cross-disciplinary spirit, which we would like to emphasize as the most important lesson to have in mind in order to make further progress in this subject.

\section{Acknowledgments}

It is a great pleasure for us to thank all the people with whom we have worked on this subject in the past, for all we have learned through our common research and for the nice atmosphere they contributed to create. We will name specially those in our group at Madrid: S. Jiménez, P.J. Pascual, M.J. Rodríguez-Plaza and Zhang Fei (Departamento de Física Teórica I, Universidad Complutense); at the Soviet Union: S.A. Gredeskul, Yu.S. Kivshar (Institute for Low Temperature Physics and Engineering, Kharkov), V.V. Konotop (Institute for Radiophysics and Electronics, Kharkov) and B. A. Malomed (P. P. Shirshov Institute for Oceanology, Moscow); at Los Alamos: A.R. Bishop, D. K. Campbell and St. Pnevmatikos (it is very regrettable that he died tragically while we were writing this review), and Guo Ben-Yu at Shanghai (University of Science and Tecnhology). We are very deeply indebted to Yuri S. Kivshar for his help with the critical reading of the manuscript. This work has been supported in part by the C.I.C. y T. (Spain) under project MAT90-0544. A. S. was also supported by fellowships from the Universidad Complutense and the Ministerio de Educación y Ciencia.

\section{References}

Abdullaev, F. Kh., A. R. Bishop and St. Pnevmatikos (eds.), 1991, Springer Proceedings in Physics: "Nonlinearity with Disorders", (Springer, Berlin-Heidelberg), in press.

Abdullaev, F. Kh., S. A. Darmanyan and M. R. Djumaev, 1989, Phys. Lett. 141A, 423. Ablowitz, M. J., D. J. Kaup, A. C. Newell and H. Segur, 1973, Phys. Rev. Lett. 30, 1262. Ablowitz, M. J., D. J. Kaup, A. C. Newell and H. Segur, 1974, Phys. Rev. Lett. 31, 125. Ablowitz, M. J. and J. R. Ladik, 1975, J. Math. Phys. 16, 598.

Ablowitz, M. J. and J. R. Ladik, 1976, J. Math. Phys. 17, 1011.

Ablowitz, M. J., M. D. Kruskal and J. R. Ladik, 1979, SIAM J. Appl. Math. 36, 478.

Afanasiev, V. V., Yu. S. Kivshar, V. V. Konotop and V. N. Serkin, 1989, Opt. Lett. 14, 805. 
Aharony, A. and J. Feder (eds.), 1989, Fractals in Physics: Essays in Honour of Benoit B. Mandetbrot, Physica 38D.

Anderson, P. W., 1958, Phys. Rev. 109, 1492.

Anker, D. and N.C. Freeman, 1978, Proc. Roy. Soc. London Ser. A 360, 592.

Aubry, S., 1975, J. Chem. Phys. 62, 3217.

Aubry, S., 1976, J. Chem. Phys. 64, 3392.

Balakrishnan, R., 1985, Physica 16D, 405.

Barabanenkov, Yu. N., Yu. A. Kratsov, S. M. Rytov and V. I. Tamarskii, 1970, Usp. Fiz. Nauk. 102, 3 [Sov. Phys. Usp. 13, 55 (1971)].

Barone, A. and G. Paternó, 1982, Physics and Applications of the Josephson Effect (Wiley, New York).

Barthes, M. and J. Léon (eds.), 1990, Lecture Notes in Physics no. 353: "Nonlinear Coherent Structures" (Springer, Berlin-Heidelberg).

Bar'yakhtar, V. G., B. A. Ivanov and A. L. Sukstankiï, 1980, Zh. Eksp. Teor. Fiz. 79, 1509 [Sov. Phys. JETP 51, 757 (1980)].

Bass, F. G., Yu. S. Kivshar, and V. V. Konotop, 1987, Zh. Eksp. Teor. Fiz. 92, 430 [Sov. Phys. JETP 65, 245 (1987)].

Bass, F. G., Yu. S. Kivshar, V. V. Konotop, and Yu. A. Sinitsyn, 1988, Phys. Reports 157, 63.

Bass, F. G., V. V. Konotop and Yu. A. Sinitsyn, 1984, Izv. Vyssh. Uchebn. Zaved., Radiofiz. 27, 718 [Sov. J. Radiophys. Elec. 27, 491 (1984)].

Bass, F. G., V. V. Konotop and Yu. A. Sinitsyn, 1985, Zh. Eksp. Teor. Fiz. 88, 541 [Sov. Phys. JETP 61, 318 (1985)].

Bass, F. G., V. V. Konotop and Yu. A. Sinitsyn, 1986, Izv. Vyssh. Uchebn. Zaved. Radiofiz. 29, 921 [Sov. J. Radiophys. Elec. 29, 708 (1987)].

Bass, F. G. and Yu. A. Sinitsyn, 1982, Izv. Vyssh. Uchebn. Zaved. Radiofiz. 25, 1302 [Sov. J. Radiophys. Elec. 25, 933 (1982)].

Bass, F. G. and A. P. Tetertov, 1986, Phys. Rep. 140, 237.

Beloshapkin, V. V., G. P. Berman and A. G. Tretyakov, 1989, Zh. Eksp. Teor. Fiz. 95, 723 [Sov. Phys. JETP 68, 410 (1989)].

Benjamin, T. B., 1967, Proc. Roy. Soc. London Ser. A, 299, 59.

Benjamin, T. B. and J. E. Feir, 1967, J. Fluid Mech. 27, 417.

Bergmann, D. J., E. Ben-Jacob, Y. Imry and K. Maki, 1983, Phys. Rev. A27, 3345.

Bernasconi, J. and T. Schneider (eds.), 1981, Physics in One Dimension, Springer Series in Solid State Sciences no. 23 (Springer, Berlin-Heidelberg).

Biller, P. and F. Petruccione, 1990, Phys. Rev. B41, 2139.

Bishop, A. R., D. K. Campbell and St. Pnevmatikos (eds.), 1989, Springer Proceedings in Physics vol. 39: "Disorder and Nonlinearity"' (Springer, Berlin-Heidelberg).

Bishop, A. R., E. Domany and J. A. Krumhans1, 1977, Ferroelectrics 16, 183.

Bishop, A. R., J. A. Krumhansl and S. E. Trullinger, 1980, Physica 1D, 1.

Bishop, A. R. and W. F. Lewis, 1978, J. Phys. C12, 3811.

Bishop, A. R. and T. Schneider (eds.), 1978, Solitons and Condensed Matter Physics, Springer Series in Solid State Sciences no. 8 (Springer, Berlin-Heidelberg).

Boesch, R., P. Stancioff and C. R. Willis, 1988, Phys. Rev. B 38, 6713.

Boesch, R. and C. R. Willis, 1989, Phys. Rev. B39, 361.

Boesch, C. R. Willis and M. El-Batanouny, 1989, Phys. Rev. B40, 2284.

Bogdan, M. M., A. M. Kosevich and V. P. Voronov, 1990, in Solitons and Applications, edited by V. G. Makhankov, V. K. Fedyanin and O. K. Pashaev (World Scientific, Singapore).

Bogdan, M. M. and A. M. Kosevich, 1990, in Solitons and Applications, edited by V. G. Makhankov, V. K. Fedyanin and O. K. Pashaev (World Scientific, Singapore). 
Bountis, T., St. Pnevmatikos and S. Protogerakis, 1990, in Lecture Notes in Physics no. 353: "Nonlinear Coherent Structures", edited by M. Barthes and J. Léon (Springer, Berlin-Heidelberg).

Bountis, T. and St. Pnevmatikos, 1990, Phys. Lett. 143A, 221.

Bourbonnais, R. and R. Maynard, 1990, Phys. Rev. Lett. 64, 1397.

Boussinesq, J., 1872, J. Math. Pures Appl. Ser. 2 17, 55.

Boyd, J., 1990, Nonlinearity 3, 177.

Braun, O. M. and Yu. S. Kivshar, 1990, Phys. Lett. 149A, 119.

Braun, O. M. and Yu. S. Kivshar, 1991, Phys. Rev. B43,1060.

Brito, R., J. A. Cuesta and A. F. Ra'ada, 1988, Phys. Lett. 128A, 360.

Bullough, R. K. and P. J. Caudrey, 1978, in Nonlinear Evolution Equations Solvable by the Inverse Spectral Transform, edited by F. Calogero (Pitman, London).

Burgers, J. M. 1974, The Nonlinear Diffusion Equation (Reidel, Dordrecht).

Campbell, D. K., J. F. Schonfeld and C. A. Wingate, 1983, Physica 9D, 1.

Campbell, D. K. and M. Peyrard, 1986, Physica 18D, 47.

Campbell, D. K., R. J. Flesch, Zhang Fei and L. Vázquez, 1991, to be published.

Caputo, J. G. and N. Flytzanis, 1990, to be published.

Caputo, J. G., A. C. Newell and M. Shelley, 1989, in Lecture Notes in Physics no. 342: "Integrable Systems and Applications", edited by M. Balabane, P. Lochak and C. Sulem (Springer, Berlin-Heidelberg).

Caputo, J. G. and A. Sánchez, 1991, to be published.

Chernov, L. A., 1960, Wave Propagation in Random Medium (McGraw-Hill, New York).

Chiao, R. I., E. Garmire and C. H. Townes, 1964, Phys. Rev. Lett. 13, 479.

Cirillo, M., R. P. Parmentier and B. Savo, 1981, Physica D3, 565.

Cole, J. D., 1959, J. Appl. Math. 9, 225.

Collins, M. A., A. Blumen, J. F. Currie, and J. Ross, 1979, Phys. Rev. B19, 3630.

Combs, J. A. and S. Yip, 1983, Phys. Rev. B28, 6873.

Combs, J. A. and S. Yip, 1984, Phys. Rev. B29, 438.

Corones, J., 1977, Phys. Rev. B16, 1763.

Currie, J. F., A. Blumen, M. A. Collins, and J. Ross, 1979, Phys. Rev. B19, 3645.

Currie J. F., S. E. Trullinger, A. R. Bishop, and J. A. Krumhansl, 1977, Phys. Rev. B15, 5567.

Dashen, R. F., B. Hasslacher and A. Neveu, 1974, Phys. Rev. D10, 4114.

Davey, A. and K. Stewartson, 1974, Proc. Roy. Soc. London Ser. A 338, 101.

Davydov, A. S., 1985, Solitons in Molecular Systems (Reidel, Dordrecht).

Delfour, M., M. Fortin and G. Payne, 1981, J. Comp. Phys. 44, 277.

Denardo, B., W. Wright, S. Putterman, and A. Larraza, 1990, Phys. Rev. Lett. 64, 1518.

Devillard, P. and B. Souillard, 1986, J. Stat. Phys. 43, 423.

De Gennes, P. G., 1966, Superconductivity of Metals and Alloys (Benjamin, New York).

Dodd, R. K., J. C. Eilbeck, J. D. Gibbon, and H. C. Morris, 1982, Solitons and Nonlinear Wave Equations (Academic press, London).

Doucot, B. and R. Rammal, 1987, Europhys. Lett. 3, 969.

Doucot, B. and R. Rammal, 1987, J. Phys. (Paris) 48, 509.

Economou, E. N., 1983, Green's Functions in Quantum Physics (Springer, BerlinHeidelberg).

Economou, E. N., 1990, Physica 167A, 215.

Efetov, K. B., 1990, in Applications of Statistical and Field Theory Methods to Condensed Matter, edited by D. Baeriswyl, A. R. Bishop and J. Carmelo (Plenum, New York).

Eilenberger, G., 1977, Z. Phys. B27, 199.

Eilenberger, G., 1981, Solitons, Springer Series in Solid State Sciences no. 19 (Springer, Berlin-Heidelberg). 
Elliot, R. J., J. A. Krumhansl and P. L. Leath, 1974, Rev. Mod. Phys. 46, 465.

Enz, V., 1964, Helv. Phys. Acta 37, 245.

Feder, J., 1988, Fractals (Plenum, New York).

Fedyanin, V. K. and V. Lisy, 1990, in Solitons and Applications, edited by V. G. Makhankov, V. K. Fedyanin and O. K. Pashaev (World Scientific, Singapore).

Fermi, E., J. R. Pasta and S. M. Ulam, 1955, Los Alamos National Laboratory Report LA-1940, 1955 (unpublished); also Collected Works of Enrico Fermi, Vol. II, p. 978 (University of Chicago Press, Chicago, 1965).

Flesch, R. J., 1987, Ph. D. Thesis, University of Southern California, unpublished.

Flesch, R. J. and S. E. Trullinger, 1987, J. Math. Phys. 28, 1619.

Flesch, R. J., M. G. Forest and A. Sinha, 1991, Physica D48, 169.

Flytzanis, N., St. Pnevmatikos and M. Remoissenet, 1985, J. Phys. C18, 4603.

Fogel, M. B., S. E. Trullinger, A. R. Bishop, and J. A. Krumhansl, 1976, Phys. Rev. Lett 36, 1411 .

Fogel, M. B., S. E. Trullinger, A. R. Bishop, and J. A. Krumhansl, 1977, Phys. Rev. B15, 1578.

Forest, M. G. and D. W. McLaughlin, 1982, J. Math. Phys. 23,1248.

Fraggis, T. and St. Pnevmatikos, 1989, in Singular Behavior and Nonlinear Dynamics (vol. 2), edited by St. Pnevmatikos, T. Bountis and Sp. Pnevmatikos (World Scientific, Singapore).

Fraggis, T., St. Pnevmatikos and E. N. Economou, 1989, Phys. Lett. 142A, 361.

Freylikher, V. D. and S. A. Gredeskul, 1991, in Progress in Optics vol. XXVIII, edited by E. Wolf, in press.

Frenkel, J. and T. R. Kontorowa, 1939, J. Phys. Sowetjunion 1, 137.

Fried, B. D. and Y. H. Ichikawa, 1973, J. Phys. Soc. Jpn. 34, 1073.

Gardner, C. S., J. M. Green, M. D. Kruskal, and R. M. Miura, 1967, Phys. Rev. Lett. 19, 1095.

Gardiner, C. W., 1983, Handbook of Stochastic Methods, Springer Series in Synergetics no. 3 (Springer, Berlin-Heidelberg).

Gefen, Y., A. Aharony and S. Alexander, 1983, Phys. Rev. Lett. 50, 77.

Goedde, C. G., A. J. Lichtenberg and M. A. Lieberman, 1990, Physica 41P, 341.

Golubov, A. A., I. L. Serpuchenko and A. V. Ustinov, 1988, Zh. Eksp. Teor. Fiz. 94, 297 [Sov. Phys. JETP 67, 1256 (1988)].

Gordon, A., 1987, Physica B146, 373.

Gordon, A., 1989, Solid State Commun. 69, 1113.

Gorentsveig, V. I., Yu. S. Kivshar, A. M. Kosevich, and E. S. Syrkin, 1990, Phys. Lett. 144A, 479.

Gredeskul, S. A. and L. A. Pastur, 1985, J. Stat. Phys. 38, 25.

Gredeskul, S. A. and Yu. S. Kivshar, 1989, Opt. Lett. 14, 1281.

Gredeskul, S. A., Yu. S. Kivshar, L. M. Maslov, A. Sánchez, and L. Vázquez, 1991, Phys. Lett. A (submitted).

Gredeskul, S. A., Yu. S. Kivshar and M. V. Yanovskaya, 1990, Phys. Rev. A41, 3994.

Griffiths, D. F., A. R. Mitchell and J. L. Morris, 1984, Comp. Meth. Appl. Mech. Eng. 45, 177.

Grüner, G. and A. Zettl, 1985, Phys. Rep. 119, 117.

Guinea, F., 1984, Phys. Rev. B30, 1884.

Guo Ben Yu, 1986, J. Comp. Math. 4, 121.

Guo Ben Yu and L. Vázquez, 1983, J. App. Sci. (China) 1, 25.

Hasegawa, A., 1981, Opt. Lett. 6, 62.

Hasegawa, A., 1988, Solitons in Optical Fibers (Springer, Berlin-Heidelberg).

Hasegawa, A. and F. D. Tappert, 1973a, Appl. Phys. Lett. 23, 142. 
Hasegawa, A. and F. D. Tappert, 1973b, Appl. Phys. Lett. 23, 171.

Havlin, S. and D. Ben-Avraham, 1987, Adv. Phys. 36, 695.

Haus, H. A. and M. N. Islam, 1985, IEEE J. Quant. Elec. 21, 1172.

Haus, J. W. and K. W. Kehr, 1987, Phys. Rep. 150, 695.

Herbst, B. M. and M. J. Ablowitz, 1989a, in Lecture Notes in Physics no. 342: "Integrable Systems and Applications", edited by M. Balabane, P. Lochak and C. Sulem (Springer, Berlin-Heidelberg).

Herbst, B. M. and M. J. Ablowitz, 1989b, Phys. Rev. Lett. 62, 2065.

Herbst, B. M., J. L. Morris and A. R. Mitchell, 1985, J. Comp. Phys. 60, 282.

Hernández-García, E., 1989, Ph. D. Thesis, Universitat des Illes Balears, unpublished.

Hernández-García, E., L. Pesquera, M. A. Rodríguez, and M. San Miguel, 1989a, J. Stat. Phys. 55, 1027.

Hernández-García, E., M. A. Rodríguez, and M. San Miguel, 1989b, Phys. Rev. B40, 9056.

Hirota, R. and K. Suzuki, 1973, Proc. IEEE 61, 1483.

Hopf, E., 1950, Commun. Pure Appl. Math 3, 201.

Ichikawa, Y. H., T. Imamura and T. Taniuti, 1972, J. Phys. Soc. Jpn. 33, 189.

Iliyinikh, A. L. and B. Ya. Shapiro, 1990, Phys. Lett. 150A, 409.

Imada, M., 1981, J. Phys. Soc. Jpn. 50, 1457.

Ivanov, B. A. and A. K. Kolezhuk, 1990, Phys. Lett. 146A, 190.

Jackiw, R. and J. R. Schrieffer, 1981, Nucl. Phys.(B190(FS3), 253.

Jiménez, S., 1990, to be published.

Jiménez, S. and L. Vázquez, 1990, Appl. Math. Comp. 35, 61.

Ji-Zhong Xu, 1990, Solid State Commun. 76, 557.

Kadomtsev, B. B. and V. I. Petviashviliī, 1970, Dokl. Akad. Nauk 192, 753 [Sov. Phys. Dokl. 15, 539, 1970].

Karpman, V. I., 1977, Pis'ma ZETF 25, 296 [JETP Lett. 25, 271 (1977)].

Karpman, V.I. and E. M. Maslov, 1977, Zh. Eksp. Teor. Fiz. 73, 537 [Sov. Phys. JETP 46 $281(1978)]$

Karpman, V. I. and E. M. Maslov, 1978, Zh. Eksp. Teor. Fiz. 75, 504 [Sov. Phys. JETP 48, 252 (1978)]

Kaup, D. J., 1976, SlAM J. Appl. Math. 31, 121.

Kaup, D. J., 1977, in The Significance of Nonlinearity in the Natural Sciences, edited by B. Kursunoglu, A. Perlmutter and L. F. Scott (Plenum, New York).

Kaup, D. J., 1983, Phys. Rev. B27, 6787.

Kaup, D. J. and E. Osman, 1986, Phys. Rev. B33, 1762.

Keener, J. P. and D. W. McLaughlin, 1977a, J. Math. Phys. 18, 2008.

Keener, J. P. and D. W. McLaughlin, 1977b, Phys. Rev. A16, 777.

Kelley, P. L., 1965, Phys. Rev. Lett. 15, 1005.

Khikmatov, N., 1982, Ph. D. Thesis, Thermophysics Department of the Uzb. S. S. R. Academy of Sciences, unpublished.

Kirsch, W., in Lecture Notes in Mathematics no. 1109: "Stochastic Aspects of Classical and Quantum Systems", edited by S. Albeverio, Ph. Combe and M. Sirugue-Collen (Springer, Berlin-Heidelberg).

Kitchenside, P. W., R. K. Bullough and P. J. Caudrey, 1978, in Solitons and Condensed Matter Physics, Springer Series in Solid State Sciences no. 8, edited by A. R. Bishop and T. Schneider (Springer, Berlin-Heidelberg).

Kivshar, Yu. S., 1991a, Phys. Rev. B43, 3493.

Kivshar, Yu. S., 199lb, in Springer Proceedings in Physics: "Nonlinearity with Disorder", edited by F. Kh. Abdullaev, A. R. Bishop and St. Pnevmatikos (Springer, BerlinHeidelberg), in press. 
Kivshar, Yu. S. and O. A. Chubykalo, 1991, Phys. Rev. B43, 5419.

Kivshar, Yu. S., S. A. Gredeskul, A. Sánchez, and L. Vázquez, 1990, Phys. Rev. Lett. 64, 1693.

Kivshar, Yu. S., S. A. Gredeskul, A. Sánchez, and L. Vázquez, 1991, Waves in Random Media, in press.

Kivshar, Yu. S., V. V. Konotop and Yu. A. Sinitsyn, 1986, Z. Phys. B. 65, 209.

Kivshar, Yu. S., V. V. Konotop and Yu. A. Sinitsyn, 1987, Izv. Vyssh. Uchebn. Zaved. Radio-fiz. 30, 374 [Sov. J. Radiophys. Elec. 30, 302 (1982)].

Kivshar, Yu. S., A. M. Kosevich and O. A. Chubykalo, 1987a, Fiz. Nizk. Temp. 13, 438 [Sov. J. Low Temp. Phys, 13, 250 (1987)].

Kivshar, Yu. S., A. M. Kosevich and O. A. Chubykalo, 1987b, Fiz. Nizk. Temp. 13, 800 [Sov. J. Low Temp. Phys. 13, 457 (1987)].

Kivshar, Yu. S., A. M. Kosevich and O. A. Chubykalo, 1987c, Zh. Eksp. Teor. Fiz. 93, 968 [Sov. Phys. JETP 66, 545 (1987)].

Kivshar, Yu. S., A. M. Kosevich and O. A. Chubykalo, 1987d, Phys. Lett. 125A, 35.

Kivshar, Yu. S., A. M. Kosevich and O. A. Chubykalo, 1988, Phys. Lett. 129A, 449.

Kivshar, Yu. S., A. M. Kosevich, O. A. Chubykalo, A. Sảnchez and L. Vázquez, 1991, Phys. Rev. B (submitted).

Kivshar, Yu. S. and B. A. Malomed, 1985, Phys. Lett 111A, 427.

Kivshar, Yu. S. and B. A. Malomed, 1988a, J. Phys. A21, 1553.

Kivshar, Yu. S. and B. A. Malomed, 1988b, Phys. Lett. 129A, 443.

Kivshar, Yu. S. and B. A. Malomed, 1989, Rev. Mod. Phys. 61, 763.

Kivshar, Yu. S. and B. A. Malomed, 1990, Phys. Rev. B42, 8561.

Kivshar, Yu. S., B. A. Malomed and A. A. Nepomnyashchiǐ, 1988, Zh. Eksp. Teor. Fiz. 94, 356 [Sov. Phys. JETP 67, 850 (1988)].

Kivshar, Yu. S. and T. K. Soboleva, 1990a, Phys. Rev. B42, 2655.

Kivshar, Yu. S. and T. K. Soboleva, 1990b, Physica B165 \&166, 1651.

Kivshar, Yu. S., Zhang Fei and L. Vázquez, 1991, Phys Rev Lett. 67, 1177.

Kjems, J. K. and M. Steiner, 1978, Phys. Rev. Lett. 41, 1137.

Knapp, R., G. Papanicolaou and B. White, 1989, in Nonlinearity and Disorder, Springer Series in Solid State Science, edited by A. R. Bishop, D. K. Campbell and St. Pnevmatikos (Springer-Verlag, Berlin).

Knuth, D. E., 1981, Seminumerical Algorithms. The Art of Computer Programming vol. 2 (Addison-Wesley, Reading).

Kodama, Y. and K. Nozaki, 1987, Opt. Lett. 12, 1038.

Koehler, T. R., A. R. Bishop, J. A. Krumhansl, and J. R. Schrieffer, 1975, Solid State Commun. 17, 1515.

Kohmoto, M., L. P. Kadanoff and C. Tang, 1983, Phys. Rev. Lett. 50, 1870.

Konotop, V. V., 1990, Phys. Lett. 146A, 50.

Konotop, V. V., A. Sánchez and L. Vázquez, 1991, Phys. Rev. B44, 2554.

Konotop, V. V. and V. E. Vekslerchik, 1991, J. Phys. A24, 767.

Korteweg, D. J. and G. de Vries, 1895, Phil. Mag. 39, 442.

Kosevich, A. M., 1990, Physica 41D, 253.

Krökel, D., K. Halas, G. Guiliani and D. Grishckow, 1988, Phys. Rev. Lett. 60, 29.

Krug, J. and H. Spohn, 1989, Europhys. Lett. 8, 219.

Krumhansl, J. A. and J. R. Schrieffer, 1975, Phys. Rev. B11, 3535.

Kunz, H. and B. Souillard, 1980, Commun. Math. Phys. 78, 201.

Kunz, C. and J. A. Combs, 1985, Phys. Rev. B31, 527.

Lakshmanan, M., 1977, Phys. Lett. 61A, 53.

Lee., P. A. and T. V. Ramakrishnan, 1985, Rev. Mod. Phys. 57, 291.

Lee, P. A., T. M. Rice and P. W. Anderson, 1974, Solid State Commun. 14, 703. 
Levy, Y. E. and B. Souillard, 1987, Europhys. Lett. 4, 233.

Lifshitz, I. M., S. A. Gredeskul and L. A. Pastur, 1988, Introduction to the Theory of Disordered Systems (Wiley, New York).

Longrenn, K. and A. C. Scott (eds.), 1978, Solitons in Action (Academic, New York).

Lorenz, E. N., 1963, J. Atmos. Sci. 20, 130.

Madhukar, A. A. and W. Post, 1977, Phys. Rev. Lett. 39, 1424.

Makhankov, V. G., 1978, Phys. Rep. 35, 1.

Makhankov, V. G. and V. K. Fedyanin, 1984, Phys. Rep. 104, 1.

Makhankov, V .G., V. K. Fedyanin and O. K. Pashaev (eds.), 1990, Solitons and Applications (World Scientific, Singapore).

Maki, K., 1978, in Solitons and Condensed Matter Physics, Springer Series in Solid State Science no. 8, edited by A. R. Bishop and T. Schneider (Springer, Berlin-Heidelberg).

Makinson, R. E. B. and A. P. Roberts, 1962, Proc. Roy. Soc. London 79, 223.

Malomed, B. A., 1985, Physica 15P, 374 (1); 385 (II).

Malomed, B. A., 1987a, Physica 27P, 113.

Malomed, B. A., 1987b, Phys. Lett 123A, 459.

Malomed, B. A., 1987c, Phys. Lett 123A, 494.

Malomed, B. A., 1988, J. Phys. C21, 5163.

Malomed, B. A., 1990a, in Solitons and Applications, edited by V. G. Makhankov, V. K. Fedyanin and O. K. Pashaev (World Scientific, Singapore).

Malomed, B. A., 1990b, Phys. Lett. 144A, 351.

Malomed, B. A., 1990c, Phys. Rev. B41, 2616.

Malomed, B. A. and A. A. Nepomnyashchili, 1991, J. Phys. Condens. Matter 3, 693.

Malomed, B. A., V. A. Oboznov and A. V. Ustinov, 1990, Zh. Eksp. Teor. Fiz. 97, 924 [Sov. Phys. JETP 70, 518 (1990)].

Malomed, B. A. and M. I. Tribelsky, 1990, Phys. Rev. B41, 11271.

Malomed, B. A. and A. V. Ustinov, 1990a, in Solitons and Applications, edited by V. G.

Makhankov, V. K. Fedyanin and O. K. Pashaev (World Scientific, Singapore

Malomed, B. A. and A. V. Ustinov, 1990b, J. Appl. Phys. 67, 3791.

Malomed, B. A. and A. V. Ustinov, 1990c, Phys. Rev. B41, 254.

Mandelbrot, B. B., 1975, Les Objects Fractal: Forme, Hasard et Dimension (Flammarion, Paris).

Mandelbrot, B. B., 1982, The Fractal Geometry of Nature (Freeman, San Francisco).

Mannella, R., 1989, in Noise in Nonlinear Dynamical Systems vol. 3: Experiments and Simulations, edited by F. Moss and P. V. E. McClinctock (Cambridge Univ. Press, New York).

Marchesoni, F. and L. Vázquez, 1985, Physica 14D, 273.

Marchesoni, F. and C. R. Willis, 1990, Europhys. Lett. 12, 491.

Matsuda, H. and K. Ishii, 1970, Prog. Theor. Phys. 45, 56.

Mattis, D. C., 1981, in Physics in One Dimension, edited by J. Bernasconi and T. Schneider, Springer Series in Solid State Sciences no. 23 (Springer, Berlin-Heidelberg).

McLaughlin, D. W. and E. A. Overman II, 1982, Phys. Rev. A26, 3497.

McLaughlin, D. W. and A. C. Scott, 1978, Phys. Rev. A18, 1652.

Mkrtchyan, G. S. and V. V. Shmidt, 1979, Solid State Commun 30, 791.

Mikeska, H. I., 1978, J. Phys. C11, L29.

Mineev, M. B., M. V. Feigel'man and V. V. Shmidt, 1981, Zh. Eksp. Teor. Fiz 81, 290 [Sov. Phys. JETP 54, 155 (1981)].

Mineev, M. B. and V. V. Shmidt, 1980, Zh. Eksp. Teor. Fiz. 79, 893 [Sov. Phys. JETP 52, 453 (1981)].

Mitchell, A. R. and R. Wait, 1977, The Finite Element Method in Partial Differential Equations (Wiley, London). 
Mitchell, A. R. and D. F. Griffiths, 1980, The Finite Difference Method in Partial Differential Equations (Wiley, London).

Mitshcke, F. M. and L. F. Mollenauer, 1987, Opt. Lett. 12, 355.

Miura, R., 1968, J. Math. Phys. 9, 1202.

Mollenauer, L. F. and K. Smith, 1988, Opt. Lett. 13, 675.

Mollenauer, L. F. and R. H. Stolen, 1984, Opt. Lett. 9, 13.

Mollenauer, L. F., R. H. Stolen and J. P. Gordon, 1980, Phys. Rev. Lett. 45, 1095.

Morse, P. and H. Feschbach, 1953, Methods of Mathematical Physics (McGraw-Hill, New York).

Moshir, M., 1981, Nucl. Phys. B185, 318.

Mott, N. F. and W. D. Twose, 1961, Adv. Phys. 10, 107.

Muroya, K., N. Saitoh and S. Watanabe, 1982, J. Phys. Soc. Jpn. 51, 1024.

Nabarro, F. R. N., 1967, Theory of Crystal Dislocations (Oxford University Press, Oxford).

Nakajima, K., T. Yamashita and Y. Onodera, 1974, J. Appl. Phys, 45, 3141.

Newell, A. C., 1985, Solitons in Mathematics and Physics (SlAM, Philadelphia).

Nozaki, K., 1982, Phys. Rev. Lett. 49, 1883.

Oboznov, V. A. and A. V. Ustinov, 1989, Phys. Lett. 139A 481.

Ostlund, S., R. Pandit, D. Rand, H. J. Schellnhuber, and E. D. Siggia, 1983, Phys. Rev. Lett. 50, 1873.

Ovchinnikov, A. A. and H. S. Erihkman, 1975, Zh. Eksp. Teor. Fiz. 67, 546 [Sov. Phys. JETP 40, 733 (1975)].

Overman II, E. A., D. W. McLaughlin and A. R. Bishop, 1986, Physica 19D, 1.

Paquet, D. and P. Leroux-Hugon, 1984, Phys. Rev. B29, 593.

Parrondo, J. M. R., M. Mañas and F. J. de la Rubia, 1990, J. Phys. A23, 2363.

Pascual, P. J. and L. Vázquez, 1985, Phys. Rev. B32, 8305.

Pascual, P. J., L. Vázquez, St. Pnevmatikos, and A. R. Bishop, 1989, in Springer Proceedings in Physics vol. 39: "Disorder and Nonlinearity, edited by A. R. Bishop, D. K. Campbell and St. Pnevmatikos (Springer, Berlin-Heidelberg).

Paul, D. I., 1978, Phys. Lett. 64A, 485.

Paul, D. I., 1979, J. Phys. C12, 585.

Perring, J. K. and T. H. R. Skyrme, 1962, Nucl. Phys. 31, 550.

Petruccione, F. and P. Biller, 1990, Phys. Rev. B41, 2145.

Peyrard, M. and M. D. Kruskal, 1984, Physica 14D, 88.

Peyrard, M. and A. R. Bishop, 1990, in Lecture Notes in Physics no. 353: "Nonlinear Coherent Structures", edited by M. Barthes and J. Léon (Springer, Berlin-Heidelberg).

Pietronero, L. (ed.), 1989, Fractals (Plenum, New York).

Pietronero, L. and E. Tosatti (eds.), 1986, Factals in Physics (North-Holland, Amsterdam).

Pillet, C. A., 1985, Commun. Math. Phys. 102, 237.

Pillet, C. A., 1986, Commun. Math. Phys. 105, 259.

Ping Sheng (ed.), 1990, Scattering and Localization of Classical Waves in Random Media (World Scientific, Singapore).

Pouget, J. and G. A. Maugin, 1984a, Physica 14D, 88.

Pouget, J. and G. A. Maugin, 1984b, Phys. Rev. B30, 5306.

Pouget, J. and G. A. Maugin, 1985a, Phys. Rev. B31, 4633.

Pouget, J. and G. A. Maugin, 1985b, Phys. Lett. 109A, 389.

Pnevmatikos, St., 1987, Phys. Lett. 122A, 249.

Pnevmatikos, St., T. Bountis and Sp. Pnevmatikos (eds.), 1989, Singular Behavior and Nonlinear Dynamics (2 vols.) (World Scientific, Singapore).

Pnevmatikos, St., N. Flytzanis and M. Remoissenet, 1986, Phys. Rev. B33, 2308.

Pnevmatikos, St., N. Flytzanis and A. R. Bishop, 1987, J. Phys. C20, 2829. 
Press, W. H., B. P. Flannery, S. A. Teukolsky, and W. T. Vetterling, 1989, Numerical Recipes (Cambridge, New York).

Pushkarov, D. I. and R. D. Atanasov, 1990, Phys. Lett. 149A,287.

Pynn, R. and T. Riste (eds.), 1987, Time-Dependent Effects in Disordered Materials (Plenum, New York).

Pynn, R. and A. Skjeltorp (eds.), 1985, Scaling Phenomena in Disordered Systems (Plenum, New York).

Qiming Li, St. Pnevmatikos, E. N. Economou, and C. M. Soukoulis, 1988a, Phys. Rev. B37, 3534

Qiming Li, C. M. Soukoulis, St. Pnevmatikos, and E. N. Economou, 1988b, Phys. Rev. B38, 11888.

Rajaraman, R., 1975, Phys. Rep. 21, 229.

Reinisch, G. and J. C. Fernandez, 1989, in Singular Behavior and Nonlinear Dynamics, edited by St. Pnevmatikos, T. Bountis and Sp. Pnevmatikos, vol. 2 (World Scientific, Singapore).

Remoissenet, M., 1986, Phys. Rev. B33, 2386.

Remoissenet, M., 1989, in Lecture Notes in Physics no. 342: "Integrable Systems and Applications", edited by M. Balabane, P. Lochak and C. Sulem (Springer, BerlinHeidelberg).

Remoissenet, M., 1990a, in Lecture Notes in Physics no. 353: "Nonlinear Coherent Structures", edited by M. Barthes and J. Léon (Springer, Berlin-Heidelberg).

Remoissenet, M., 1990b, in Proceedings of the Les Houches Winter School in Theoretical Physics on Partially Integrable Nonlinear Equations and their Physical Applications (Reidel, Dordrecht).

Rice, M. J., 1979, Phys. Lett. 71A, 153.

Rice, M. J., A. R. Bishop, J. A. Krumhansl, and S. E. Trullinger, 1976, Phys. Rev. Lett. 36, 432.

Rice, M. J. and E. J. Mele, 1980, Solid State Commun. 35,487.

Rice, M. J. and J. Timonen, 1979, Phys. Lett. 73A, 3698.

Richtmyer, R. D. and K. W. Morton, 1967, Difference Methods for Initial Value Problems (Wiley-Interscience, New York).

Risken, H., 1984, The Fokker-Planck Equation, Springer Series in Synergetics no. 18 (Springer, Berlin-Heidelberg).

Rodriguez-Plaza, M. J. and L. Vázquez, 1988, in Mathematics + Physics, Lectures on Recent Results no. 3, edited by L. Streit (World Scientific, Singapore)

Rodríguez-Plaza, M. J. and L. Vázquez, 1990, Phys. Rev. B41, 11437.

Rubinstein, J., 1970, J. Math. Phys. 11, 258.

Sakai, S., M. R. Samuelsen and O. H. Olsen, 1987, Phys. Rev. B36, 217.

Salerno, M., E. Joergensen and M. R. Samuelsen, 1984, Phys. Rev. B30, 2635.

Sánchez, A. and L. Vázquez, 1989, in Singular Behavior and Nonlinear Dynamics edited by St. Pnevmatikos, T. Bountis and Sp. Pnevmatikos, vol. 2 (World Scientific, Singapore).

Sánchez, A. and L. Vázquez, 1991, Phys. Lett. 152A, 184.

Sánchez, A., L. Vázquez and V. V. Konotop, 1991, Phys. Rev. A44, 1086.

Sanz-Serna, J. M. and J. G. Verwer, 1986, IMA J. Num. Anal. 6, 25.

Schneider, T. and E. Stoll, 1978, Phys. Rev. B17, 1302.

Scott Russell, J., 1844, Proc. Roy. Soc. Edinburgh, 319.

Scott, A. C., 1969, Am. J. Phys. 37, 52.

Scott, A. C., 1990, in the preface of Lecture Notes in Physics no. 353: "Nonlinear Coherent Structures", edited by M. Barthes and J. Léon (Springer, Berlin-Heidelberg).

Scott, A. C., F. Y. F. Chu and D. W. McLaughlin, 1973, Proc. IEEE 61, 1443. 
Scott, A. C., F. Y. F. Chu and S. A. Reible, 1976, J. Appl. Phys. 47, 3272.

Serpuchenko, I. L. and A. V. Ustinov, 1987, Pis'ma ZETF 46, 435 [JETP Lett. 46, 549 (1987)].

Shimizu, K. and Y. H. Ichikawa, 1972, J. Phys. Soc. Jpn. 33, 789.

Shivamoggi, B. K., 1988, Introduction to Nonlinear Fluid-Plasma Waves (Kluwer Academic, New York).

Sinai, Ya. G., 1987, J. Stat. Phys. 46, 861.

Spatschek, K. H., 1987, Fortschr. Phys. 35, 491.

Stancioff, P., C. R. Willis, M. El-Batanouny and S. Burdick, 1986, Phys. Rev. B33, 1904.

Stanley, H. E. and P. Meakin, 1988, Nature 335, 405.

Stanley, H. E. and N. Ostrowsky (eds.), 1985, On Growth and Form (Martinus Nijhoff, Dordrecht).

Steinhardt, P. J. and S. Ostlund, 1987, The Physics of Quasicrystals (World Scientific, Singapore).

Strauss, W. and L. Vázquez, 1978, J. Comp. Phys. 28, 271.

Strauss, W., 1989, Nonlinear Wave Equations, Regional Conference Series in Mathematics no. 73 (Amer. Math. Soc., Providence).

Taha, T. R. and M. J. Ablowitz, 1984, J. Comp. Phys. 55, 203

Taha, T. R. and M. J. Ablowitz, 1988, J. Comp. Phys. 75, 540.

Taki, M., K. H. Spatschek, J. C. Fernandez, R. Grauer and G. Reinisch, 1989, Physica 40D, 65.

Tappert, F. D. and C. M. Varma, 1970, Phys. Rev. Lett. 25, 1108.

Theodorakopoulos, N., 1979, Z. Phys. B33, 385.

Thouless, D. J., 1974, Phys. Rep. 13, 93.

Thouless, D. J., 1980, Phys. Rep. 67, 5.

Toda, M., 1967, J. Phys. Soc. Jpn. 22, 431.

Trefethen, L. N., 1982, SIAM Review 24, 113.

Trefethen, L. N., 1982, Commun. Pure Appl. Math. 37, 289.

Tsurui, K., 1977, Prog. Theor. Phys. 48, 1196.

Ustinov, A. V., 1989, Phys. Lett. 136A, 155.

van Kampen, N., 1981, Stochastic Processes in Physics and Chemistry (North-Holland, Amsterdam)

Vázquez, L., 1989, in Symmetries in Science III, edited by B. Gruber and F. Iachello (Plenum, New York).

Vicsek, T., 1989, Fractal Growth Phenomena (World Scientific, Singapore).

Vysloukh, V. A., I. V. Ivanov and A. V. Cherechik, 1987, Izv. Vyssh. Uchebn. Zaved. Radiofiz. 30, 980 [Sov. J. Radiophys. Elec. 30, 674 (1984)].

Wada, Y. and J. R. Schrieffer, 1978, Phys. Rev. B18, 3897.

Wadati, M., 1983, J. Phys. Soc. Jpn. 52, 2642.

Wadati, M. and Y. Akutsu, 1984, J. Phys. Soc. Jpn. 53, 3342.

Wadati, M., 1990, to be published.

Weiner, A. M, J. P. Heritage, R. J. Hawkins, R. N. Thurston, E. M. Kirschner and W. J. Tomlinson, 1988, Phys. Rev. Lett. 61, 2445.

West, B. J., 1985, Lecture Notes in Biomathematics no. 62: “An Essay on the Importance of Being Nonlinear" (Springer, Berlin-Heidelberg)

Willis, C. R., 1989, Phys. Rev. B39, 7067.

Willis, C. R., M. El-Batanouny and P. Stancioff, 1986 Phys. Rev. B33, 1904.

Whitham, G. B., 1974, Linear and Nonlinear Waves (Wiley, New York)

Wu, J., J. Wheatley, S. Putterman and I. Rudnick, 1987, Phys. Rev. Lett. 59, 2774.

Yajima, N. and J. Satsuma, 1979, Prog. Theor. Phys. 62, 370.

Zabusky, N. J., 1981, J. Comp. Phys. 43, 195. 
Zabusky, N. J. and M. D. Kruskal, 1965, Phys. Rev. Lett. 15, 240.

Zakharov, V. E., S. V. Manakov, S. P. Novikov and L. P. Pitaevsky, 1984, Theory of Solitons (Consultants Bureau, New York) (Russian Edition, 1980, Nauka, Moscow).

Zakharov, V. E. and A. B. Shabat, 1971, Zh. Eksp. Teor. Fiz. 61, 118 [Sov. Phys. JETP 34, 62 (1972)].

Zakharov, V. E. and A. B. Shabat, 1973, Zh. Eksp. Teor. Fiz. 64, 1627 [Sov. Phys. JETP 37, 823 (1972)].

Zettl, A., 1988, in Methods and Applications of Nonlinear Dynamics, edited by A. W. Sáenz (World Scientific, Singapore).

Ziman, J. M., 1979, Models of Disorder (Cambridge University Press, Cambridge).

Zvezdin, A. K., 1979, Pis'ma ZETF 29, 605 [JETP Lett. 29, 553 (1979)]. 